\title{
A Cross-Platform SpMV Framework on Many-Core Architectures
}

\author{
YUNQUAN ZHANG and SHIGANG LI, State Key Laboratory of Computer Architecture, \\ Institute of Computing Technologies, Chinese Academy of Sciences \\ SHENGEN YAN, SenseTime Group Limited, Department of Information Engineering, \\ Chinese University of Hong Kong \\ HUIYANG ZHOU, Department of Electrical and Computer Engineering, \\ North Carolina State University
}

\begin{abstract}
Sparse Matrix-Vector multiplication (SpMV) is a key operation in engineering and scientific computing. Although the previous work has shown impressive progress in optimizing SpMV on many-core architectures, load imbalance and high memory bandwidth remain the critical performance bottlenecks. We present our novel solutions to these problems, for both GPUs and Intel MIC many-core architectures. First, we devise a new SpMV format, called Blocked Compressed Common Coordinate (BCCOO). BCCOO extends the blocked Common Coordinate (COO) by using bit flags to store the row indices to alleviate the bandwidth problem. We further improve this format by partitioning the matrix into vertical slices for better data locality. Then, to address the load imbalance problem, we propose a highly efficient matrix-based segmented sum/scan algorithm for SpMV, which eliminates global synchronization. At last, we introduce an autotuning framework to choose optimization parameters. Experimental results show that our proposed framework has a significant advantage over the existing SpMV libraries. In single precision, our proposed scheme outperforms clSpMV COCKTAIL format by $255 \%$ on average on AMD FirePro W8000, and outperforms CUSPARSE V7.0 by $73.7 \%$ on average and outperforms CSR5 by $53.6 \%$ on average on GeForce Titan X; in double precision, our proposed scheme outperforms CUSPARSE V7.0 by $34.0 \%$ on average and outperforms CSR5 by $16.2 \%$ on average on Tesla K20, and has equivalent performance compared with CSR5 on Intel MIC.
\end{abstract}

This work was supported by the National Natural Science Foundation of China under Grant No. 61502450, Grant No. 61432018, Grant No. 61521092, and Grant No. 61272136; National Key Research and Development Program of China under Grant No.2016YFB0200803; NSF project 1216569; and a gift fund from AMD Inc. Extension of Conference Paper. The additional contributions of this article over the previously published work of S. Yan et al. at PPoPP-2014 include the following:

(1) This article extends our proposed BCCOO format for Intel Xeon Phi processors by introducing innerblock transpose (Section 3.2.2).

(2) We propose a new segmented sum/scan strategy for Intel Xeon Phi processors to explore the potential performance of their 512-bit SIMD instructions (Section 3.2.2).

(3) We present the experimental results on Intel Xeon Phi processor, and compare the performance with NVIDIA and AMD GPUs in the context of yaSpMV (Section 6).

(4) We also extend the yaSpMV library to support double precision (Section 3.2.3) and present the experimental results in Section 6.

The new material is more than one third of our PPoPP-2014 paper.

Authors' addresses: Y. Zhang, State Key Laboratory of Computer Architecture, Institute of Computing Technologies, Chinese Academy of Sciences, Beijing 100190, China; email: yunquan.cas@gmail.com; S. Li (corresponding author), State Key Laboratory of Computer Architecture, Institute of Computing Technologies, Chinese Academy of Sciences, Beijing 100190, China; email: shigangli.cs@gmail.com; S. Yan (corresponding author), SenseTime Group Limited, Department of Information Engineering, The Chinese University of Hong Kong; email: yanshengen@gmail.com; H. Zhou, Department of Electrical and Computer Engineering, North Carolina State University, Raleigh, NC; email: hzhou@ncsu.edu.

Permission to make digital or hard copies of part or all of this work for personal or classroom use is granted without fee provided that copies are not made or distributed for profit or commercial advantage and that copies show this notice on the first page or initial screen of a display along with the full citation. Copyrights for components of this work owned by others than ACM must be honored. Abstracting with credit is permitted. To copy otherwise, to republish, to post on servers, to redistribute to lists, or to use any component of this work in other works requires prior specific permission and/or a fee. Permissions may be requested from Publications Dept., ACM, Inc., 2 Penn Plaza, Suite 701, New York, NY 10121-0701 USA, fax +1 (212) 869-0481, or permissions@acm.org.

(c) 2016 ACM 1544-3566/2016/10-ART33 $\$ 15.00$

DOI: http://dx.doi.org/10.1145/2994148 
CCS Concepts: - Computing methodologies $\rightarrow$ Parallel algorithms; $\bullet$ Computer systems organization $\rightarrow$ Parallel architectures

Additional Key Words and Phrases: SpMV, segmented scan, BCCOO, OpenCL, CUDA, GPU, Intel MIC, parallel algorithms

ACM Reference Format:

Yunquan Zhang, Shigang Li, Shengen Yan, and Huiyang Zhou. 2016. A cross-platform SpMV framework on many-core architectures. ACM Trans. Archit. Code Optim. 13, 4, Article 33 (October 2016), 25 pages.

DOI: http://dx.doi.org/10.1145/2994148

\section{INTRODUCTION}

Sparse Matrix-Vector Multiplication (SpMV) is a key linear algebra algorithm and is heavily used in many important application domains. Many-core architectures feature high computational throughput and memory access bandwidth, which are the promising targets to accelerate the workloads like SpMV. According to the granularity of each core, many-core architectures can be divided into two categories. The first is based on massive light cores, like NVIDIA and AMD GPUs, and high throughput is achieved by massive fine-grained parallelism. The second is based on many heavy cores, in which both coarse-grained and fine-grained parallelism are supported. Intel Many Integrated Core (Intel MIC) architecture belongs to the second category. This article aims at proposing a high performance SpMV framework for these two kinds of many-core architectures.

Although the sequential implementation of SpMV is straightforward, its parallel implementation is quite challenging, especially on many-core architectures. First, since the nonzeros in a matrix may not be evenly distributed across different rows, the rowbased parallelization usually suffers from the load imbalance problem. This problem is more severe on GPU architectures, since the threads operate in the Single-Instruction Multiple-Thread (SIMT) manner and the execution time is literally determined by the slowest thread. Secondly, SpMV puts high pressure on the memory hierarchy. The matrix data exhibit poor data reuse, as each nonzero element is only used once for computing the corresponding dot product. Besides, the access pattern of the multiplied vector is irregular, due to the discontinuous locations of the nonzeros in each row. On GPUs, memory coalescing [Ueng et al. 2008], namely, all the threads in a warp access the consecutive memory address, is a key factor to achieve high memory bandwidth. However, irregular accesses will destroy memory coalescing, which makes the memory accesses serialized.

There has been a lot of research on accelerating SpMV by many-core processors. To reduce the memory footprint size and fully exploit the performance of the manycore architectures, researchers have proposed a bunch of many-core-oriented storage formats, such as COO [Bell and Garland 2009], ELLPACK [Bell and Garland 2009], ELL-R [Vázquez et al. 2011], SELL [Monakov et al. 2010], BCSR and BELL [Choi et al. 2010], ESB [Liu et al. 2013], and SELL-C- $\sigma$ [Kreutzer et al. 2014]. On the other hand, the Compressed Sparse Row (CSR) format is still dominant on traditional CPU architectures because of high performance and good compression. Recent research work [Daga and Greathouse 2015; Liu and Schmidt 2015; Greathouse and Daga 2014] proposed new algorithms for CSR-based SpMV, which attempted to make the CSR format also achieve high performance on many-core architectures. Furthermore, given the different features of target hardware platforms and different characteristics of sparse matrices, offline autotuning or benchmarking [Choi et al. 2010; Li et al. 2015] is commonly used to improve the performance. Although previous work has achieved impressive performance improvement for SpMV, load imbalance and high memory bandwidth requirement remain the fundamental performance bottlenecks of SpMV. 
In this article, we propose our novel solution to SpMV. Since the proposed solution is yet another SpMV framework, we name it as yaSpMV. We first propose a new format for sparse matrices to alleviate the memory bandwidth pressure. Our new format is referred to as Blocked Compressed Common Coordinate (BCCOO), as it is built upon the Common Coordinate (COO) format. The BCCOO format extends the $\mathrm{COO}$ format with blocking to reduce the size for both row and column index arrays. Then, it uses bit flags to drastically reduce the size of the row index array. To improve the data locality of the multiplied vector, we partition the sparse matrix into vertical slices and align the slices in a top-down manner. Such vertically partitioned BCCOO is referred to as the $\mathrm{BCCOO}+$ format.

To address the load imbalance problem, we design a new highly optimized segmented scan/sum kernel for SpMV. In our approach, each thread processes the same number of consecutive nonzero blocks and performs sequential segmented scans/sums to generate partial sum results. Then, each workgroup/thread block will run the parallel segmented scan on the last partial sum results. When the final dot-product results require accumulating partial sums across multiple workgroups/thread blocks, adjacent synchronization [Yan et al. 2013] is used to eliminate the overhead of global synchronization. To further improve the performance of our SpMV kernel, we also introduce an autotuning framework to explore optimization parameters for different sparse matrices and different platforms. The parameters to be tuned form a large search space. We prune the search space of the parameters using some heuristics and reduce the autotuning time to a few seconds.

The yaSpMV framework is implemented based on OpenCL [Stone et al. 2010], which supports general purpose parallel programming on heterogeneous computing platforms, including GPUs and Intel MIC. Experimental results show that our proposed single format fits nearly all of the 20 sparse matrices used in the experiments. In single precision, compared with the vendor-tuned library CUSPARSE V7.0, our proposed scheme achieves $73.7 \%$ on average on GeForce Titan X; compared with the clSpMV [Su and Keutzer 2012], which combines advantages of many existing formats, our proposed scheme achieves up to $195 \%$ and $70 \%$ on average on GTX680 GPUs, and up to $2617 \%$ and $255 \%$ on average on AMD FirePro W8000 GPUs; compared with CSR5 [Liu and Vinter 2015], our proposed scheme achieves a performance gain of 53.6\% on average on GeForce Titan X, and $14.9 \%$ on average on AMD FirePro W8000. In double precision, our proposed scheme outperforms CUSPARSE V7.0 by $34.0 \%$ on average on Tesla K20, and outperforms CSR5 by $16.2 \%$ on average on Tesla K20, and by $9.7 \%$ on average on AMD FirePro W8000. On Intel MIC, our proposed scheme has almost equivalent performance compared with CSR5.

The remainder of this article is organized as follows. Section 2 presents our proposed BCCOO/BCCOO+ format for sparse matrices. Section 3 details our proposed customized matrix-based segmented scan/sum approach for SpMV. Section 4 summarizes our autotuning framework. The experimental methodology and the results are discussed in Sections 5 and 6, respectively. Section 7 addresses the related work. Section 8 concludes the article.

\section{THE BCCOO FORMAT}

Our proposed BCCOO format builds upon the COO format. In this section, we first present the $\mathrm{COO}$ format as the background, and then introduce the BCCOO format and its extension-BCCOO+. We will use the sparse matrix in Figure 1 as an example.

\subsection{COO Format}

The COO format is a widely used format for sparse matrices. It has explicit storage for the column and row indices for all nonzeros in a sparse matrix. For example, the 


$$
\mathrm{A}=\left[\begin{array}{llllllll}
0 & 0 & a & 0 & 0 & 0 & b & c \\
0 & 0 & d & e & 0 & 0 & f & 0 \\
0 & 0 & 0 & 0 & g & h & i & j \\
k & l & 0 & 0 & m & n & o & p
\end{array}\right]
$$

Fig. 1. An example of a sparse matrix.

$$
\begin{aligned}
& \text { Row_index }=\left[\begin{array}{llllllllllllllll}
0 & 0 & 0 & 1 & 1 & 1 & 2 & 2 & 2 & 2 & 3 & 3 & 3 & 3 & 3 & 3
\end{array}\right] \\
& \text { Col_index }=\left[\begin{array}{llllllllllllllll}
2 & 6 & 7 & 2 & 3 & 6 & 4 & 5 & 6 & 7 & 0 & 1 & 4 & 5 & 6 & 7
\end{array}\right]
\end{aligned}
$$

\begin{tabular}{|c|}
\hline Row_index $=\left[\begin{array}{lllll}0 & 0 & 1 & 1 & 1\end{array}\right]$ \\
\hline Col_index $=\left[\begin{array}{lllll}1 & 3 & 0 & 2 & 3\end{array}\right]$ \\
\hline Value $\left.=\left(\begin{array}{llllllllll}{[a} & 0 & b & c & 0 & 0 & g & h & i & j\end{array}\right]\right)$ \\
\hline
\end{tabular}

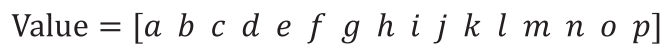

Fig. 2. The $\mathrm{COO}$ format of matrix A.

Fig. 3. The blocked $\mathrm{COO}$ format of matrix A with the block size of $2 \times 2$.

matrix in Figure 1 can be represented with a row index array, a column index array, and a data value array, as shown in Figure 2.

The parallelization strategy suitable with COO is segmented scan/reduction [Bell and Garland 2009]. As highlighted in Bell and Garland [2009] and Su and Keutzer [2012], the advantage of the COO format is that it does not suffer from the load imbalance problem, and can achieve consistent performance over different types of sparse matrices. However, the key problem of the COO format is that it needs to explicitly store both the row index and the column index for every nonzero element. Therefore, it has the worst memory footprint [Su and Keutzer 2012].

\subsection{BCCOO Format}

The BCCOO format extends the COO format in two ways. First, we combine the blockbased design with the COO format. In block-based formats, such as blocked ELLPACK and blocked CSR [Choi et al. 2010], one block of data values will share the same row index and the same column index. Therefore, the storage overhead of the row index array and the column index array can be significantly reduced. Figure 3 shows the blocked COO (BCOO) format of the matrix A in Figure 1 with the block size of $2 \times 2$.

From Figure 3, we can see that there are five nonzero blocks. Both the row index array and the column index array have been reduced significantly. The first nonzero $2 \times 2$ block is $\left(\begin{array}{ll}a & 0 \\ d & e\end{array}\right)$, and its block-based row index and column index are 0 and 1 , respectively. The next nonzero $2 \times 2$ block is $\left(\begin{array}{ll}b & c \\ f & 0\end{array}\right)$, and its blocked-based row index and column index are 0 and 3, respectively. Note that in Figure 3, we use two arrays rather than a single array to store the data value. For a block size with the height larger than 1, we put different rows in different arrays, such that both the row index and column index can be used directly to index the data in each of the value arrays. Such data arrangement is also helpful for contiguous memory accesses. The same as all the block-based formats, the BCOO format may contain zero elements even in a nonzero block. 


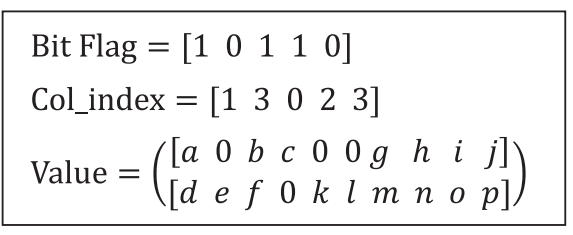

Fig. 4. The BCCOO format of matrix A with the block size of $2 \times 2$.

Our key extension to the BCOO format is to use a bit flag array to compress the row index array. We first calculate the difference value of each pair of the adjacent elements in the row index array. If the difference value is not greater than 1 , we set the corresponding bit in the bit flag array to the difference value. If the difference value is greater than 1, we set a corresponding number of 1's in the bit flag array. Then, we flip the bits of 1's and 0's in the bit flag array, such that a bit value of "0" represents a row stop, namely, the corresponding block is the last nonzero block in the row. A bit value of " 1 " represents that the corresponding block is not the last nonzero block in a row. When calculating the partial sums, this representation can eliminate the row stop check for each nonzero block (see Section 3.2 for details). The row index information can be reconstructed from the bit flag array by accumulating the number of row stops. Thus, the row index array is compressed in a lossless manner. We refer to this format as BCCOO. For matrix A in Figure 1, the BCCOO format with the block size of $2 \times 2$ is shown in Figure 4.

Compared with the BCOO format shown in Figure 3, the column index array and the data value arrays remain the same. The row index array becomes a bit vector of 5 bits. Assuming that integers are used for row indices of BCOO format, BCCOO achieves a compression ratio of 32 for the row index array. In order to remove the control flow to check the end of the bit flag array, we pad it with bit " 1 ," such that the length of the bit flag array is a multiple of the number of nonzero blocks processed by a workgroup.

Similar to row-index arrays, we also try to reduce the data transmission overhead for the column index arrays using difference functions. First, we logically partition the column index array into multiple segments, each of which is corresponding to the working set (i.e., the total nonzero blocks to be processed) of a thread. Then, we use a difference function on each segment of the column index array. In this way, there is no interthread dependency when reconstructing the column indices. The resulting difference value array is stored using the short data type instead of the regular integer type. If a difference value is beyond the range of a signed short, we replace it with a fixed value "-1," which means that the original column index array needs to be accessed for this particular index.

\subsection{BCCOO+ Format}

We also propose an extension to our BCCOO format to improve the locality of the accesses to the multiplied vector, referred to as the $\mathrm{BCCOO}+$ format. In this format, we first partition a sparse matrix into vertical slices, and then align the slices in a top-down manner. Next, we apply the BCCOO format on the vertically sliced and rearranged matrix. However, the column index array is generated based on the block coordinates in the original matrix, rather than the transformed matrix. This is because we need the original column indices to locate the corresponding elements in the multiplied vector for dot-product operations. For matrix A in Figure 1, the vertically sliced and rearranged matrix becomes matrix $\mathrm{B}$ in Figure 5(a), in which the number of slices is 2 and the slice width is 4 . The BCCOO+ format of $\mathrm{A}$ is shown in Figure 5(b) with the block size of $2 \times 2$. As shown in Figure 5 , the bit flag array encodes that there is only one 


$$
\mathrm{B}=\left[\begin{array}{llll}
0 & 0 & a & 0 \\
0 & 0 & d & e \\
0 & 0 & 0 & 0 \\
k & l & 0 & 0 \\
0 & 0 & b & c \\
0 & 0 & f & 0 \\
g & h & i & j \\
m & n & o & p
\end{array}\right]
$$

(a) The vertically sliced and rearranged ma rix of matrix $\mathrm{A}$.

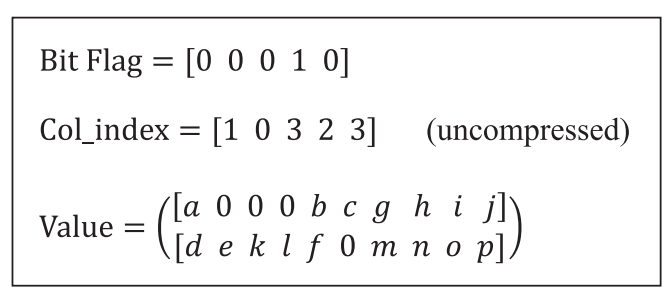

(b) The bit flag, column index, and data value arrays.

Fig. 5. The BCCOO+ format of matrix A in Figure 1.

$$
\mathrm{A} * \vec{y}=\left[\begin{array}{cccc}
0 & 0 & a & 0 \\
0 & 0 & d & e \\
0 & 0 & 0 & 0 \\
k & l & 0 & 0
\end{array}\right] *\left[\begin{array}{l}
y[0] \\
y[1] \\
y[2] \\
y[3]
\end{array}\right]+\left[\begin{array}{cccc}
0 & 0 & b & c \\
0 & 0 & f & 0 \\
g & h & i & j \\
m & n & o & p
\end{array}\right] *\left[\begin{array}{l}
y[4] \\
y[5] \\
y[6] \\
y[7]
\end{array}\right]
$$

Fig. 6. Matrix-vector multiplication as a sum of the products between its vertical slices and the corresponding vector segments.

nonzero block in row 0 , row 1 , and row 2 , and two nonzero blocks in row 3 . The column indices of these blocks, however, are determined from matrix A rather than matrix B. Taking the $2 \times 2$ block $\left(\begin{array}{c}g h \\ m n\end{array}\right)$ as an example, its column index value indicates that it resides at column 2 in matrix $\mathrm{A}$.

The benefit of $\mathrm{BCCOO}+$ format can be illustrated by the matrix-vector multiplication between matrix A and vector $\vec{y}$, namely, $\mathrm{A}^{*} \vec{y}$. Different rows in the same vertical slice use the same segment of $\vec{y}$ to compute the dot product. Thus, the temporal locality of vector $\vec{y}$ is improved by BCCOO+. However, since the BCCOO+format breaks the original matrix into slices, the intermediate results of each slice need to be combined to generate the final results. For matrix A in Figure 1, the computation of $A^{*} \vec{y}$ based on the BCCOO+ format is shown in Figure 6. We can see that it is necessary to use a temporary buffer to store the intermediate results, and invoke an additional kernel to combine them. Suppose the original matrix is divided into $s$ slices and the length of $\vec{y}$ is $l$. The size of the temporary buffer is calculated by $s * l *$ sizeof(datatype). Extra memory overhead hurts the performance. Thus, the BCCOO+format is not always preferred over the $\mathrm{BCCOO}$ format. We resort to autotuning to determine either the $\mathrm{BCCOO}$ or $\mathrm{BCCOO}+$ format should be used.

\subsection{Auxiliary Information for SpMV}

To facilitate the computation of SpMV, the following information is computed and stored along with the BCCOO/BCCOO+ format. First, based on the number of nonzeros that each thread will process, we compute the location of the first result generated by each thread, namely, the row index that the result belongs to. We use matrix $\mathrm{C}$ in Figure 7(a) as an example, in which each element represents a data block. To simplify the discussion, we assume the block size is $n \times 1$. As discussed in Section 2.2, for a block size with the height larger than 1 , each row will be stored in a separate value array. The $\mathrm{BCCOO}$ format of matrix $\mathrm{C}$ is shown in Figure 7(b). Assuming each thread processes four nonzero blocks, we will compute the row index of the first result generated by each thread. Such information can be computed by a scan operation on the bitwise inverse of the bit flag array in the BCCOO format. In this example, thread 0 processes the first four nonzero data blocks $A^{\prime}, B^{\prime}, C^{\prime}$, and $D^{\prime}$. The first computation result, namely, 


$$
\mathrm{C}=\left[\begin{array}{cccccccc}
A^{\prime} & 0 & B^{\prime} & 0 & C^{\prime} & 0 & D^{\prime} & E^{\prime} \\
0 & 0 & 0 & F^{\prime} & 0 & 0 & G^{\prime} & 0 \\
0 & H^{\prime} & 0 & I^{\prime} & 0 & J^{\prime} & 0 & 0 \\
0 & K^{\prime} & L^{\prime} & M^{\prime} & 0 & N^{\prime} & O^{\prime} & P^{\prime}
\end{array}\right]
$$

(a) Sparse Matrix C.

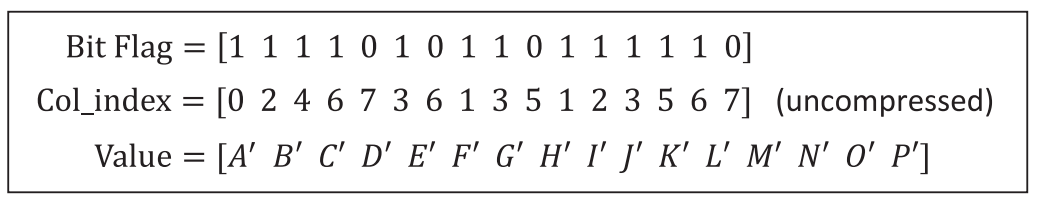

(b) The BCCOO format of Matrix C.

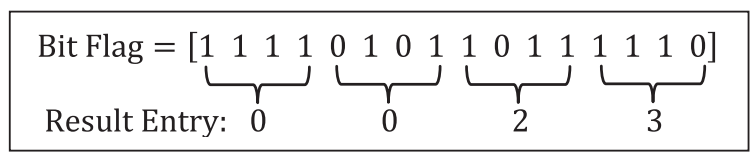

(c) The location of the first result generated by each thread. Assume there are four threads and each thread processes four nonzero blocks.

Fig. 7. Auxiliary information for SpMV.

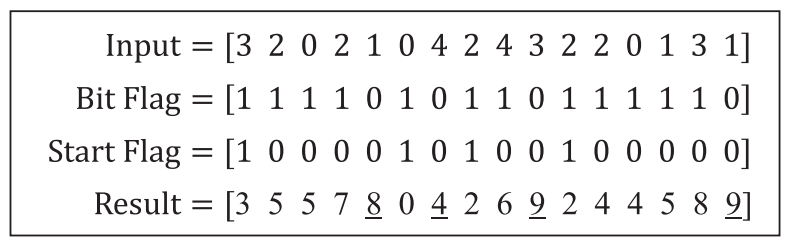

Fig. 8. An inclusive segmented scan with the start flags generated from the bit flag array in Figure 7(b).

$A^{\prime *} y[0]$, is part of the final result for the dot product between row 0 and the multiplied vector. Thus, the result entry for thread 0 is set to 0 . Similarly, thread 1 processes the next four nonzero blocks $E^{\prime}, F^{\prime}, G^{\prime}$, and $H^{\prime}$. As block $E^{\prime}$ still belongs to row 0 , the result entry for thread 1 is also set to 0 .

\section{EFFICIENT MATRIX-BASED SEGMENTED SCAN/SUM FOR SPMV}

For a sparse matrix stored in our BCCOO/BCCOO+ format, SpMV can be implemented in three logical steps: (1) read the data value arrays and multiply them with the corresponding vector values indexed by the Col_index array; (2) perform a customized matrix-based segmented scan/sum using the bit flag array; and (3) combine the partial results, and write back the final results to global memory using the result entry array. In our proposed scheme, all three of these steps are implemented in a single kernel so as to minimize the kernel invocation overhead.

\subsection{Segmented Scans}

The segmented scan primitive scans multiple data segments that are stored together. A start flag array is typically used to identify the first element of a segment. We show an example of the inclusive segmented scan in Figure 8. Its start flag array is generated from the bit flag array of the BCCOO format in Figure 7. The output of the inclusive segment scan is the Result array in Figure 8. Note that for SpMV, the complete segmented scan results are not necessary. Actually, we only need the last sum of each segment, which is marked with underscores in the Result array. Thus, a more 


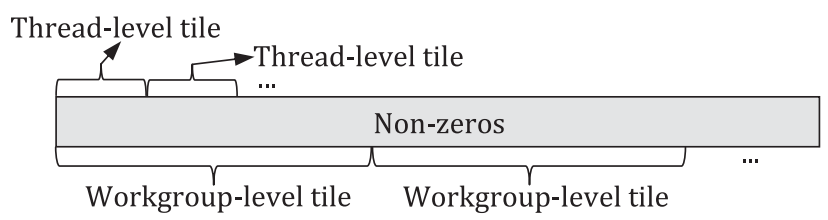

Fig. 9. Even workload distribution: each workgroup/thread block works on a workgroup-level tile; each thread works on a thread-level tile of nonzero blocks.

lightweight segmented sum can be used for SpMV. However, considering that a warp is the minimum scheduling unit for GPUs, segmented sum has almost equal computation to segmented scan when there are few nonzeros in each row. Besides, for segmented sum, it has to check whether the corresponding bit for each nonzero value is a row stop or not, which brings overhead. Thus, we consider both segmented scan and segmented sum to implement SpMV.

Two main approaches have been proposed to parallelize the segmented scan primitive on GPUs. One is a tree-based approach [Blelloch 1989], which builds a binary tree through different processing stages. The tree-based approach suffers from the load imbalance problem. Furthermore, it requires workgroup-level synchronization between stages as discussed in Dotsenko et al. [2008]. The other is a matrix-based approach [Dotsenko et al. 2008], which is proposed to improve memory efficiency and overcome the load imbalance problem. Our proposed BCCOO/BCCOO+ format suits better with the matrix-based segmented scan and we further customize it for SpMV.

\subsection{Customized Matrix-Based Segmented Scan/Sum for SpMV}

3.2.1. Per-Thread and Per-Workgroup Working Sets. In our segmented sum/scan approach for SpMV, the nonzero blocks, the bit flag array, and the column index array are divided evenly among workgroups. The working set of each workgroup is referred to as a workgroup-level tile, which in turn will be divided evenly among the threads within the workgroup. The working set of a thread is referred to as a thread-level tile, as shown in Figure 9. For a thread-level tile, a single/few load(s) from the bit flag array (such as loading a single short type of data) will be sufficient to provide all the bit flag information. Compared with the previous approaches, which load the row index information for every nonzero, significant bandwidth will be saved.

Since a thread-level tile may contain row stops, each thread will write its last partial sum into a temporary array, called last_partial_sums. Then, a parallel segmented scan [Sengupta et al. 2007] will be performed on this last_partial_sums array. The start flags of the last_partial_sums array are generated by each thread. We further perform a quick check to see whether we can skip the parallel segmented scan operation at the workgroup level. It is the case when each thread in a workgroup encounters a row stop, which results in the segment size being 1 for the parallel segmented scan. When the nonzeros in a row span multiple workgroups/thread blocks, we leverage the recently proposed adjacent synchronization [Yan et al. 2013] for interworkgroup communication, which eliminates global synchronization.

3.2.2. Computing the Partial Sums and the Final Results. We design three strategies to compute the intraworkgroup partial sums and get the final results. The first and the second strategies are designed for GPUs, and the third one is proposed especially for Inte MIC.

Strategy (1): In the first strategy, each thread has an array, called intermediate sums, to keep all the intermediate sums of its thread-level tile. This intermediate_sums array can be stored in shared memory (also called local memory in OpenCL), registers, or split between shared memory and registers. This strategy works well if most rows in a 


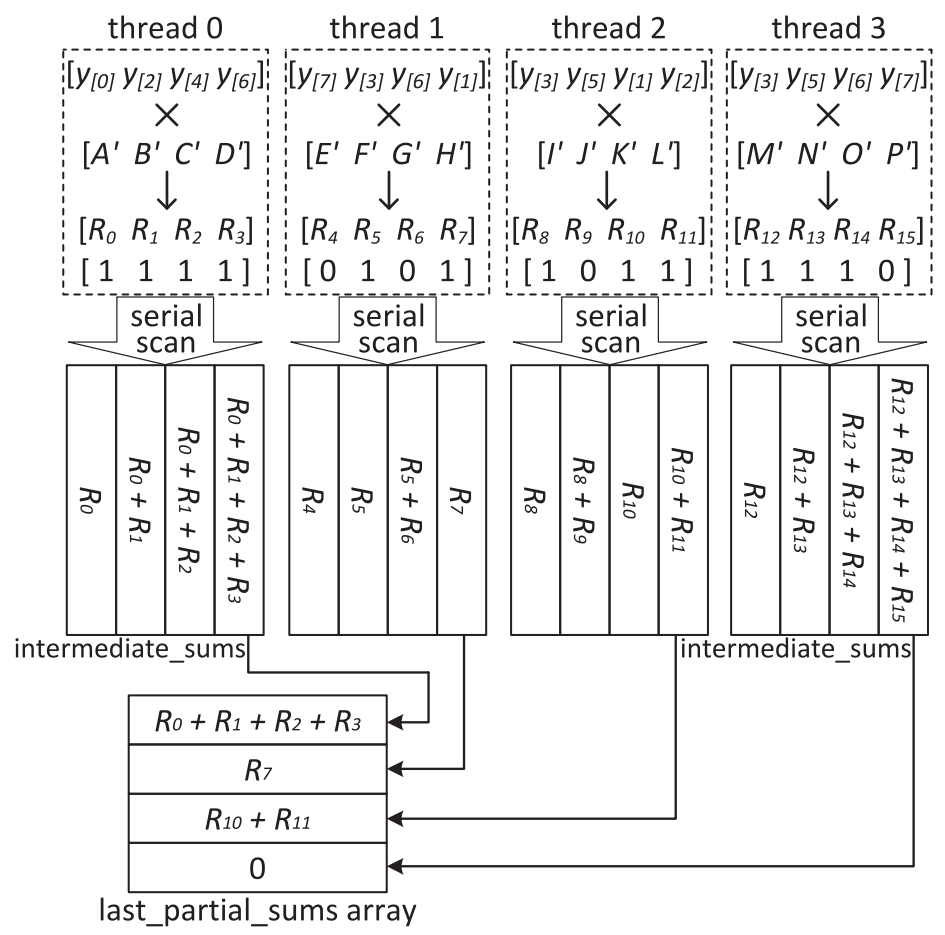

Fig. 10. Computing segmented scans: strategy (1), which uses per-thread buffers, namely, the intermediate_sums arrays to store the intermediate sum results.

sparse matrix have very few nonzeros. For the matrix $\mathrm{C}$ in Figure 7(a), the computation of the intraworkgroup partial sums is illustrated in Figure 10, in which we assume that each thread-level tile contains four nonzero blocks and there are four threads in a workgroup. Each thread performs a sequential segmented scan, and stores the results in its intermediate_sums array. Each thread uses the last partial sum to update the corresponding entry of the last_partial_sums array, which locates in the shared memory and can be accessed by all the threads in a workgroup. If the last element of a thread-level tile is a row stop, the last partial sum of this thread is 0 , as shown by thread 3 in Figure 10.

Memory coalescing [Ueng et al. 2008] is the key factor to achieve high bandwidth when accessing the data value array. We view the data value array as a twodimensional array with the width as the thread-level tile size. Then, with a transpose operation, the threads in a warp will access the data in a row-by-row manner, thereby satisfying the memory coalescing requirement. We do the same thing to the Col_index array. The transpose operation can be done either online or offline. With an online approach, the threads in a warp read one tile at a time in a coalesced manner and multiply with the corresponding vector elements, then store the results in a shared memory buffer still in the row-based manner. Later on, when performing the segmented scan, the threads read the buffer in a column-based manner. If nonzeros in a row are close to each other, online transpose may achieve better performance due to the improved locality for the multiplied vector. We use online transpose for our first strategy. For offline transpose, the two-dimensional data value array is previously transposed during the format generation phase. Different from online transpose, offline transpose does 
not require a shared memory buffer for transposition. We will use offline transpose in strategy (2).

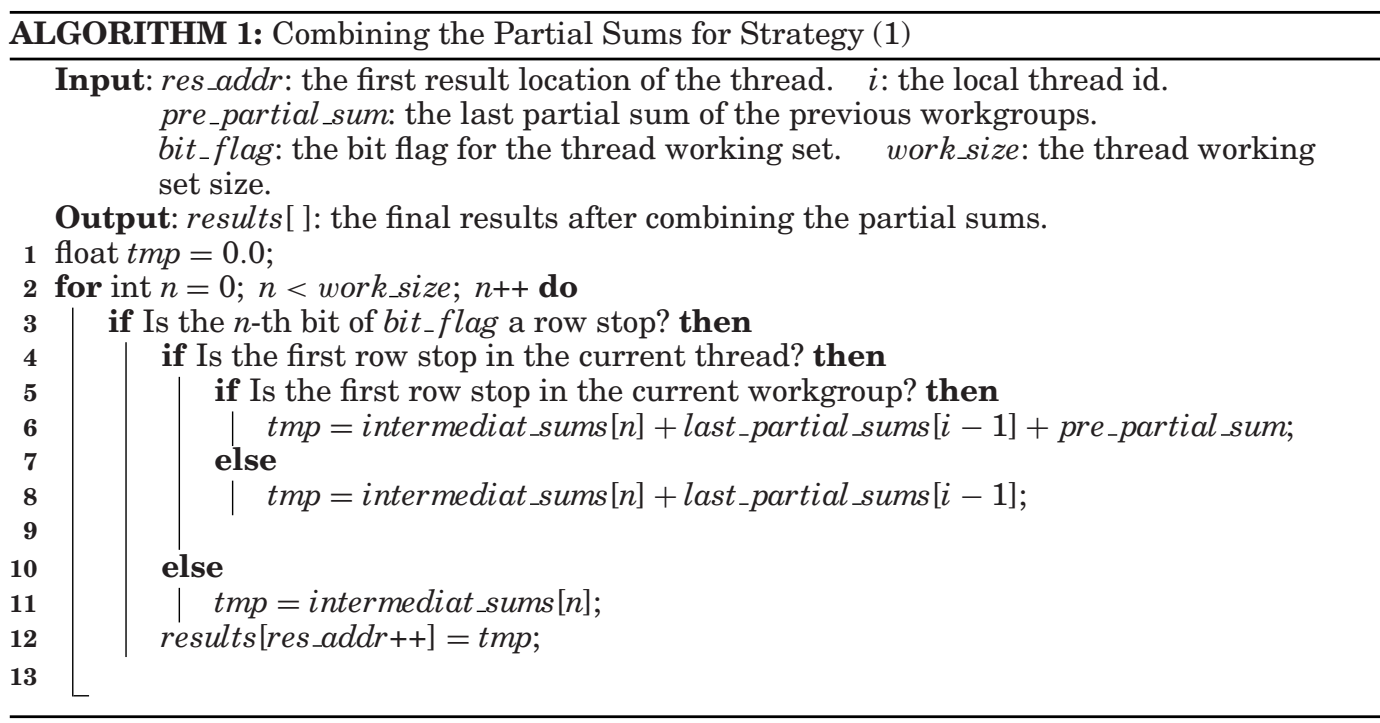

Next, we need to combine the results in the per-thread intermediate_sums arrays, the results in the per-workgroup last_partial_sums array, and also the results from other workgroups to generate the final output of SpMV. Algorithm 1 illustrates how to generate the final output for strategy (1). Each thread will go through its intermediate_sums array. For each element, it checks whether the corresponding bit flag is a row stop (line 3). If not, it means the corresponding result has already been incorporated into the sum of the segment. For a row stop, a thread further checks whether it is the first stop in its thread-level tile (line 4). If not, it means the thread-level tile contains the complete segment and the corresponding result is the final result (line 11). In the example shown in Figure 10, for thread 1, the entry in its intermediate_sums array containing $(R 5+R 6)$ is such a case. If a row stop is the first in a thread-level tile (such as the entry containing $R 4$ for thread 1 in Figure 10), there are two possibilities. One is that the segment spans multiple threads within a workgroup (lines 7 and 8). Then, the last_partial_sums array of the workgroup will be used to retrieve the last partial sum of the previous threads. For example, the entry containing $(R 0+R 1+R 2+R 3)$ in the last_partial_sums array will be added to $R 4$ of thread 1 in Figure 10. The other possibility is that the segment spans multiple threads across workgroups (lines 5 and 6). In this case, we also need to accumulate the last partial sum results of the previous workgroups. We resort to adjacent synchronization to avoid global synchronization as discussed in Section 3.2.3. At last, the final results are written back to global memory (line 12).

Strategy (2): In our second strategy, we allocate a result cache in shared memory to only store the sum of each segment. This strategy works better for long segments and also benefits from efficient memory writes, as we can store the result cache to global memory in a coalesced way. With this strategy, the offline transpose is used to ensure coalesced memory reads from the data value array and the Colindex array. After performing the multiplication with vector elements, each thread carries out a segmented sum sequentially on its thread-level tile, using the bit flag array as the 


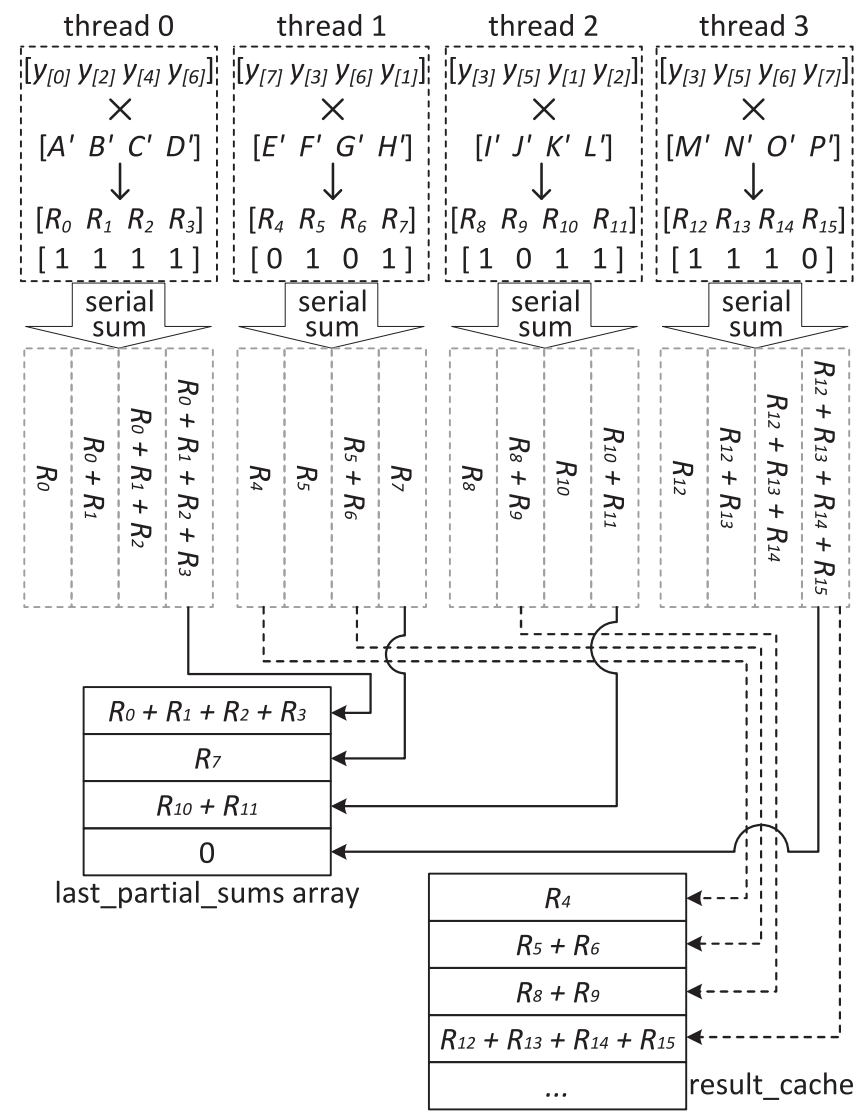

Fig. 11. Computing segmented sum: strategy (2), which uses a per-workgroup result cache to store segmented sums. The dashed blocks mean that the intermediate sums are not stored.

mask for the segments. All the segmented sums will be written to the result cache with the help of the result entry information generated along with the BCCOO format.

Using the matrix $\mathrm{C}$ in Figure 7 (a) as an example, strategy (2) is illustrated in Figure 11. We assume that each thread-level tile contains four nonzero blocks and there are four threads in a workgroup. The result entry information shown in Figure 7 is used for updating the result cache. For example, as shown in Figure 7, the result entry for thread 1 and thread 2 is 0 and 2, respectively. Therefore, when thread 1 encounters the first row stop, it uses its current sum $R 4$ to update the entry 0 of the result cache. When thread 1 encounters the second row stop, it uses the sum $R 5+R 6$ to update the entry 1 of the result cache. In a sense, the result entry information partitions the result cache among different threads in a workgroup. When the number of row stops in a workgroup-level tile is larger than the result cache size, the extra segmented sums will be stored in the result array in global memory, which will be reaccessed later to generate the final outputs.

The same as the first strategy, each thread also writes its last partial sum to the last_partial_sums array. To generate the start flags for the last_partial_sums array, in either strategy, each thread simply checks whether its bit flags contain a 0 (namely, a row stop). If so, its last partial sum should be a start for a segment in the last_partial_sums array. For the examples in Figures 10 and 11, the start flags are $[0,1,1,1]$, since all threads except thread 0 process a tile containing a row stop. After updating the 
last_partial_sums array, all the threads in the workgroup perform a parallel segmented scan [Sengupta et al. 2007] on the last_partial_sums array using the start flags. In our example in Figure 10 or Figure 11, this parallel scan can be skipped since all the segment sizes are 1.

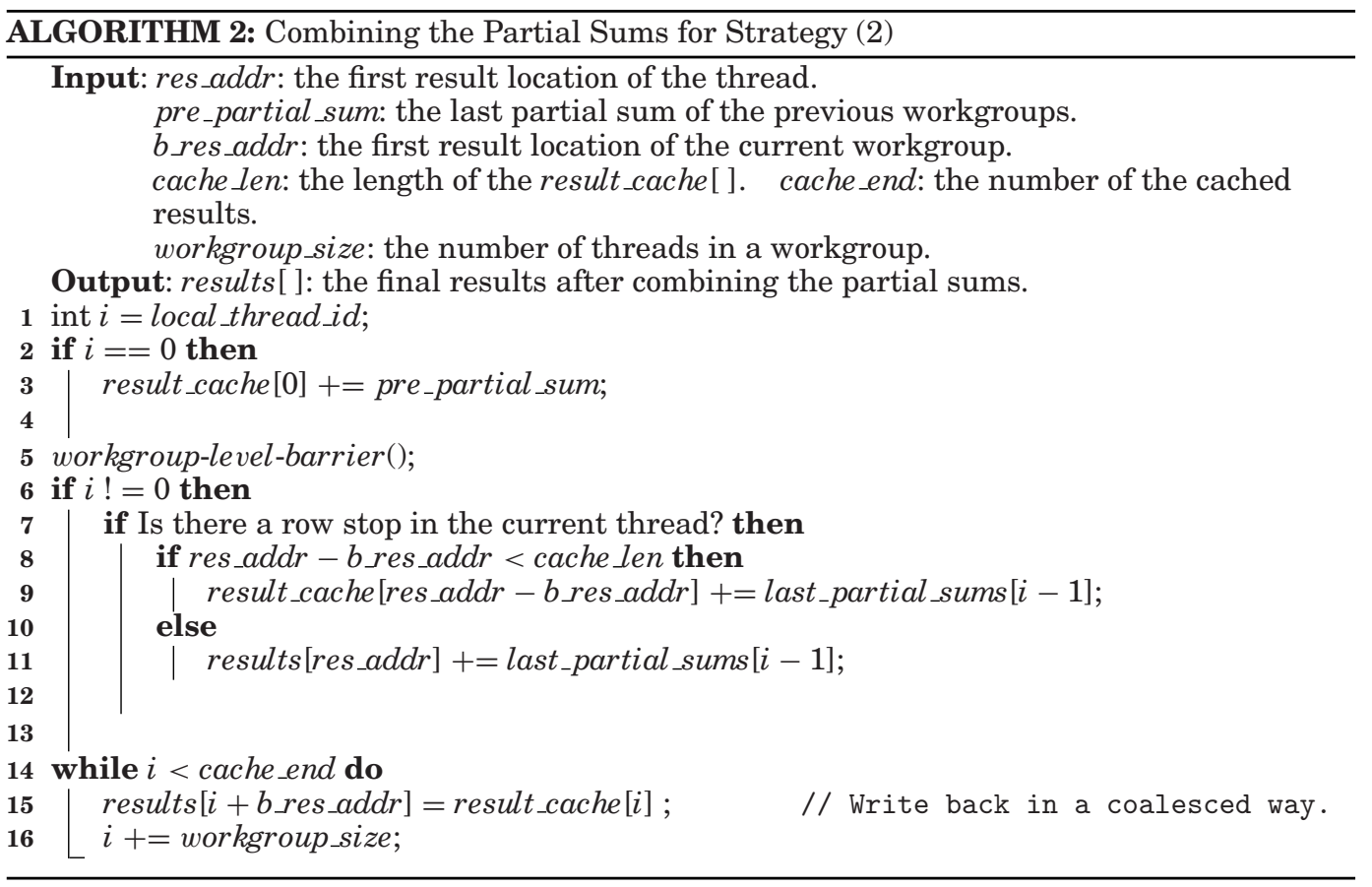

Algorithm 2 illustrates how to generate the final output for strategy (2). In strategy (2), there are no per-thread intermediate sum arrays. Instead, there is a per-workgroup result cache. For thread 0 , it updates the entry 0 of the result cache with the last partial sum from the previous workgroup (lines 2-4). To avoid data race at the entry 0 of the result cache, a workgroup-level synchronization is used (line 5). Each thread except thread 0 first checks whether there are row stops in its thread-level tile (line 7). If so, it means that the thread has generated some partial sums corresponding to the row stops. Each thread only needs to process the partial sum at the first row stop (such as $R 4$ in the result cache in Figure 11). For subsequent row stops in the thread, the partial sums in the result cache are already the complete segment sums. Next, each thread except thread 0 adds the last partial sum from the previous thread to the partial sum at the first row stop, which is stored either in result cache (lines 8 and 9 ) or in global memory (lines 10 and 11 ). For example, $R 0+R 1+R 2+R 3$ from the last_partial_sums array is added to $R 4$ in the result cache in Figure 11. After the result cache is updated, it is written back to global memory in a memory-coalescing way (lines 14-16).

Strategy (3): Intel MIC and GPUs have different hardware architectures and different performance optimization tricks. Thus, we propose the third strategy specifically for MIC, as illustrated in Figure 12. To achieve high throughput on MIC based on OpenCL, two key points should be noticed. First the local memory in OpenCL, which is usually used as a high performance shared buffer, has no corresponding hardware implementation in MIC. The data in local memory is actually put in the global memory of MIC, with extra software overhead. As illustrated in Figure 12, the intermediate 


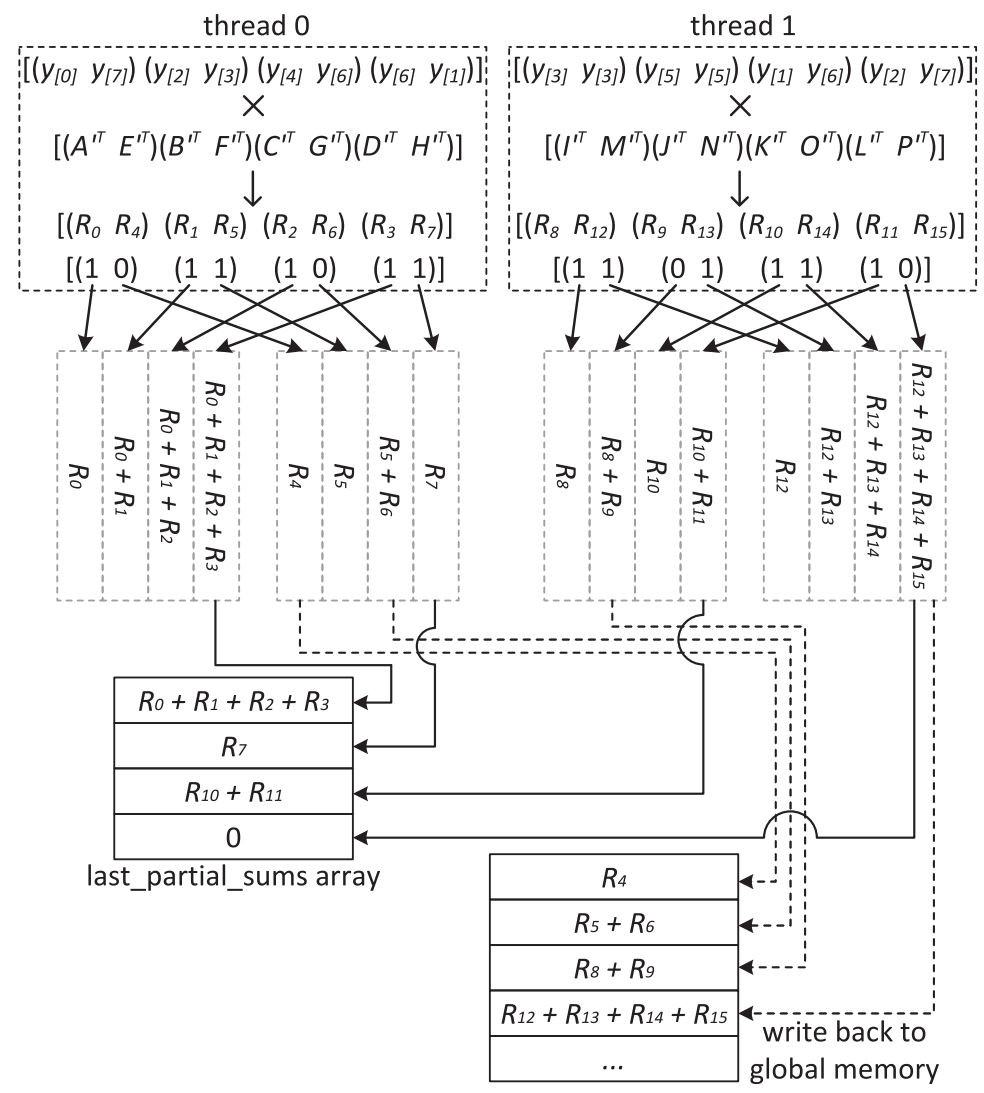

Fig. 12. Computing segmented sum: strategy (3), proposed only for Intel MIC. The dashed blocks mean that the intermediate sums are not stored.

sums are not stored, and therefore the local memory consumption is avoided. All the segmented sums will be written back to the global memory directly with the help of the result entry information. Besides, the last_partial_sums array is also stored in the global memory.

Secondly, the 512-bit SIMD instruction in MIC should be fully utilized. To achieve this, besides the offline transpose for the data value array used in strategy (2), we conduct another inner-block transpose for the BCCOO format. In Figure 12, $A^{\prime T}$ means the transposed block of $A^{\prime}$. After the inner-block transpose, each thread can execute both the multiplication and addition operations in SpMV in a SIMD manner. The width of the SIMD instruction is decided by the width of each block (after transposition). When the width of each block is small, the potential performance of the 512-bit SIMD instruction cannot be fully exploited. However, enlarging the width of each block will bring more zero elements, which reduces the sparse matrix compression efficiency. To solve this dilemma, we propose a method of workload coalescing. As shown in Figure 12, the workload of two threads in Figure 11 is coalesced to be done by one thread. Take thread 0 in Figure 12 as an example; thread 0 processes two nonzero blocks (i.e., $A^{\prime T}$ and $E^{\prime T}$ ) simultaneously, and thus the width of SIMD is doubled. The number of threads, whose workload is coalesced to be done by one thread, is called bunch_size in the following content. 


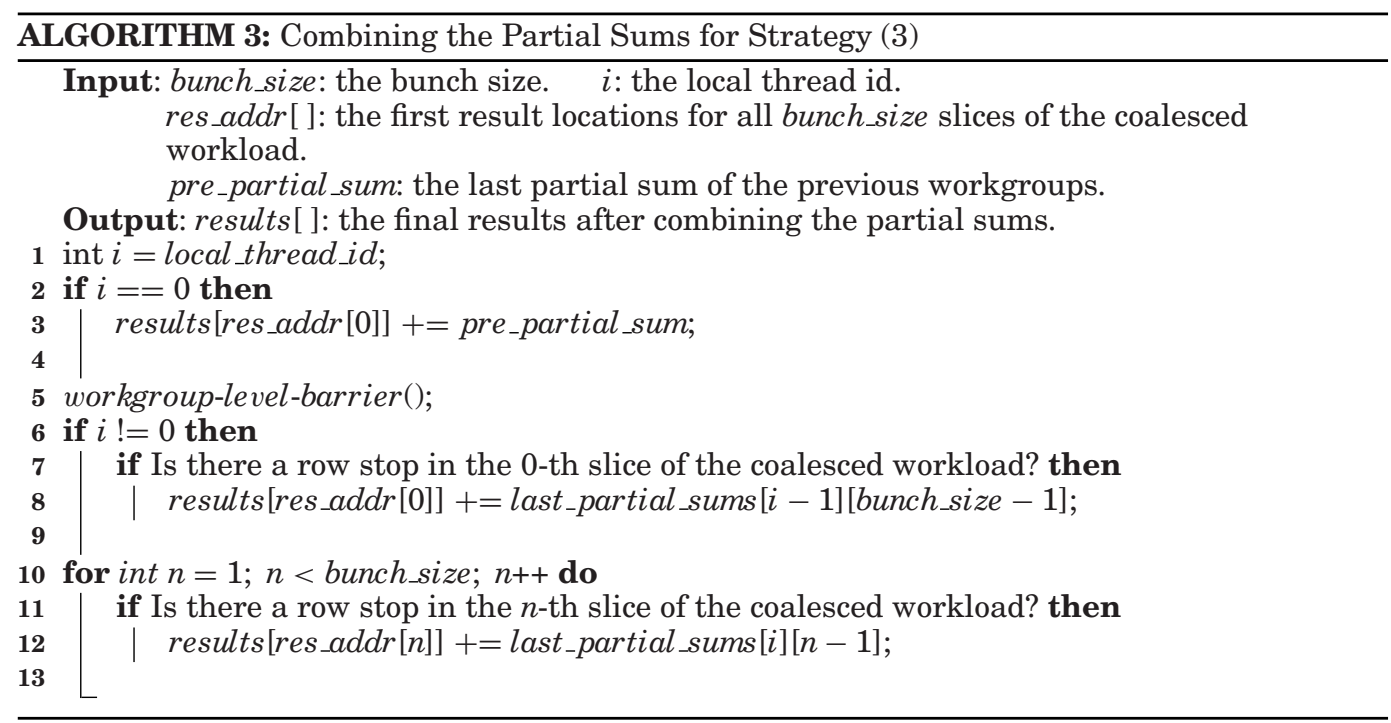

Algorithm 3 illustrates how to generate the final output for strategy (3). In strategy (3), the intermediate results are written back to the global memory directly without being cached. Recall that there are total bunch_size slices of workload coalesced to be done by one thread. For thread 0, it updates the entry 0 of the result cache with the last partial sum from the previous workgroup (lines 2-4). Each thread except thread 0 first checks whether there are row stops in its 0 -th slice of the coalesced workload. If so, the thread accumulates the last patrial sum (the partial sum of the (bunch_size-1)-th slice workload of the last thread) to the corresponding result entry in global memory (lines 7 and 8 ). For example, $R 0+R 1+R 2+R 3$ from the last_partial_sums array is added to $R 4$ in the global memory in Figure 12. Then, the current thread checks whether there are row stops in the $n$-th slice workload of its own, where $n \in[1$,bunch_size-1]. If so, the current thread accumulates the partial sum of the (n-1)-th slice to the first result location of the $n$-th slice (lines 11 and 12).

3.2.3. Accumulating Partial Sums Across Workgroups. As discussed in Section 3.2.2, for segments spanning multiple workgroups, the partial sums should be accumulated across the workgroups. The last workgroup, which contains the row stop, needs to accumulate the partial sums of previous workgroups. Here, we make an implicit assumption that the workgroup-level tiles are distributed to workgroups in order. In other words, workgroup 0 processes the first tile, workgroup 1 processes the second tile, etc. The current GPUs dispatch workgroups in order. Therefore, we can directly use the workgroup ids in the kernel. If a GPU dispatches workgroups out of order, workgroups can get such logic workgroup ids from global memory using atomic fetch-and-add operations. This approach incurs small performance overhead, less than $2 \%$ in our experiments. We use a global memory array Grp_sum to accumulate partial sums across workgroups. We use another initialized Sync_flag array, whose elements have one-to-one correspondence to Grp_sum elements, for synchronization. Workgroup 0 updates the first entry Grp_sum[0] with its last partial sum, and then updates the corresponding element in the $S y n c_{-}$flag array. For a subsequent workgroup with id $X$, if it does not contain a row stop, it waits for the entry $S y n c_{-} f l a g[X-1]$ to be updated by workgroup $(X-1)$, and then updates Grp_sum[X] with the sum of its last partial sum and Grp_sum[X-1]. If a workgroup contains a row stop, it breaks these chained updates and directly updates 
Table I. Tunable Parameters of the Autotuning Framework on GPUs

\begin{tabular}{|c|c|c|}
\hline \multicolumn{2}{|c|}{ Parameter Name } & Possible Values \\
\hline \multicolumn{2}{|c|}{ Matrix format } & BCCOO, BCCOO+ \\
\hline \multicolumn{2}{|c|}{ Col_index compress } & Yes, No \\
\hline \multicolumn{2}{|c|}{ Block width } & $1,2,4$ \\
\hline \multicolumn{2}{|c|}{ Block height } & $1,2,3,4$ \\
\hline \multicolumn{2}{|c|}{ Data type for the bit flag array } & $\begin{array}{l}\text { Unsigned char, unsigned } \\
\text { short, unsigned int }\end{array}$ \\
\hline \multicolumn{2}{|c|}{ Vertical slice number } & $1,2,4,8,16,32$ \\
\hline \multicolumn{2}{|c|}{ Transpose } & Offline, online \\
\hline \multicolumn{2}{|c|}{ Texture memory for multiplied vector } & Yes, No \\
\hline \multicolumn{2}{|c|}{ Workgroup size } & $64,128,256,512$ \\
\hline \multirow[t]{2}{*}{$\begin{array}{l}\text { Strategy } \\
1\end{array}$} & $\begin{array}{l}\text { Registers for the per-thread } \\
\text { intermediate sums array } \\
\text { (Reg_size) }\end{array}$ & $0,8,16,32$ \\
\hline & $\begin{array}{l}\text { Shared memory for the per- } \\
\text { thread intermediate sums array } \\
\text { (ShM_size) }\end{array}$ & $0,8,16,32$ \\
\hline \multirow{2}{*}{$\begin{array}{l}\text { Strategy } \\
2\end{array}$} & Thread-level tile size & $8,16,24,32,40,64,96,128$ \\
\hline & $\begin{array}{l}\text { Result cache size (multiple of } \\
\text { the workgroup size) }\end{array}$ & $1,2,3,4$ \\
\hline
\end{tabular}

Grp_sum $[X]$ with its last partial sum. This approach is called adjacent synchronization [Yan et al. 2013].

However, to use the approach of adjacent synchronization on some platforms (such as AMD GPUs) with cache enabled by default, atomic primitives have to be used to guarantee that each workgroup can access the latest values of $S y n c_{-}$flag $[X-1]$ and Grp_sum $[X-1]$ once being updated by the previous workgroup. For single precision, we use the atomic primitive atomic_xchg. However, there is no corresponding atomic primitives that directly support double precision. By enabling $c l \_k h r \_i n t 64 \_b a s e \_a t o m i c s$, we can use the 64-bit atomic primitive atom_xchg, which only supports the types of long and ulong. Using the function as_ulong(), we treat the values and variables of double type as ulong type, which can then be used in atom_xchg. So far, we can use the atomic primitive on double-precision variables and values for adjacent synchronization without losing precision.

\section{AUTOTUNING FRAMEWORK}

As discussed in Sections 2 and 3, we propose BCCOO and BCCOO+ formats for sparse matrices, and three new strategies to compute segmented sums/scans for SpMV. To find the optimal solution for a sparse matrix, we build an offline autotuning framework to select the format, the computing strategy, and their associated parameters. Then, the OpenCL code is generated according to the selected parameters from this autotuning framework. We also use this framework to exploit the texture cache for the multiplied vector in single precision on GPUs. Another optimization is that we use the "unsigned short" data type for the colindex array if the width of a sparse matrix is less than 65535. In this case, there is no need to further compress the col_index array using the approach discussed in Section 2.2. The parameters that this framework explores for GPUs and MIC are listed in Table I and Table II, respectively. Note that when strategy (1) is used to compute the segmented scan, the thread-level tile size is the size of the immediate_sums array, which is the sum of the parameters, Reg_size and ShM_size. 
Table II. Tunable Parameters of the Autotuning Framework on MIC

\begin{tabular}{|l|l|}
\hline Parameter Name & Possible Values \\
\hline \hline Matrix format & BCCOO \\
\hline Col_index compress & Yes, No \\
\hline Block width & $1,2,4$ \\
\hline Block height & $1,2,4$ \\
\hline Data type for the bit flag array & $\begin{array}{l}\text { Unsigned char, unsigned } \\
\text { short, unsigned int }\end{array}$ \\
\hline Workgroup size & $1,2,4,8,16,32$ \\
\hline Thread-level tile size & $32 \sim 1024$ \\
\hline Bunch size & $1,2,4$ \\
\hline
\end{tabular}

Besides, the strategy (3), which is proposed specifically for Intel MIC, includes bunch size as a tuning parameter.

As shown in Table I and Table II, there are many parameters to tune, which form a relatively large search space. Although the framework mainly aims at iterative SpMV, we try to minimize the overhead of offline autotuning using the following optimizations. First, we use GPUs to accelerate the translation from the COO format to the $\mathrm{BCCOO} / \mathrm{BCCOO}+$ format. Second, we cache compiled kernels in a hash table so that they can be reused for different matrices. Third, we prune the search space using the following heuristics: (1) Since the memory footprint is highly dependent on block dimensions, we only select the block dimensions with the top four minimum memory footprints; (2) we always use the texture memory for the multiplied vector in single precision, and always use offline transpose; (3) we reduce the searching range of the result cache size for the second strategy; (4) we set the shared memory size as 0 for the per-thread intermediate sums array for the first strategy; (5) we use the BCCOO+ format only when the width of the sparse matrix is larger than the height; (6) we reduce the searching range of the thread-level tile size according to the dimensions of the sparse matrix. With these optimizations, our autotuning framework only runs 28 iterations of SpMV on average for the 20 sparse matrices in our study (shown in Table III). The average autotuning time is 3.6 seconds on average for the 20 sparse matrices on a machine with an $\operatorname{Intel}(\mathrm{R}) \operatorname{Xeon}(\mathrm{R}) \mathrm{E} 5-2660$ @ $2.20 \mathrm{GHz}$ (only one core is used) and an NVIDIA GTX680 GPU. Compared with the optimal results obtained from an exhaustive search of the parameters listed in Table I, our autotuning results are identical to the optimal ones on NVIDIA GTX680 and AMD W8000 GPUs. On NVIDIA GTX480, however, the optimal configurations show $10.5 \%$ better performance for the matrix Epidemiology, which prefers no texture memory usage, and $11.1 \%$ better performance for the matrix Circuit, which prefers online transpose. As we can expect, a finer grain parameter selection may further improve the performance. In addition, we find that block width, block height, thread-level tile size, and bunch size are the parameters that top affect the performance among all the parameters.

\section{EXPERIMENTAL METHODOLOGY}

Our experiments have been performed on six different platforms: Nvidia GTX680, Nvidia GTX480 GPU, Tesla K20, GeForce Titan X, AMD FirePro W8000, and Intel MIC SE10P. We use a total of 20 sparse matrices for performance evaluation. Table III summarizes the information of the sparse matrices. These matrices have been widely used in previous works [Bell and Garland 2009; Choi et al. 2010; Monakov et al. 2010; Su and Keutzer 2012; Liu and Vinter 2015; Williams et al. 2009]. All matrices except Dense are downloadable at the University of Florida Sparse Matrix Collection [Davis and $\mathrm{Hu}$ 2011]. In our experiments, we also use CUSPARSE V7.0 [NVIDIA 2014], CUSP 
Table III. The Sparse Matrices used in the Experiments

\begin{tabular}{|c|c|c|c|c|}
\hline Spyplot & Name & Size & Non-zeros (NNZ) & NNZ/Row \\
\hline & Dense & $2 \mathrm{~K}^{*} 2 \mathrm{~K}$ & 4000000 & 2000 \\
\hline & Protein & $36 \mathrm{~K}^{*} 36 \mathrm{~K}$ & 4344765 & 119 \\
\hline & FEM/Spheres & $83 \mathrm{~K} * 83 \mathrm{~K}$ & 6010480 & 72 \\
\hline & FEM/Cantilever & $62 \mathrm{~K} * 62 \mathrm{~K}$ & 4007383 & 65 \\
\hline & Wind Tunnel & $218 \mathrm{~K}^{*} 218 \mathrm{~K}$ & 11634424 & 53 \\
\hline & FEM/Harbor & $47 \mathrm{~K}^{*} 47 \mathrm{~K}$ & 2374001 & 59 \\
\hline & QCD & $49 \mathrm{~K}^{*} 49 \mathrm{~K}$ & 1916928 & 39 \\
\hline & FEM/Ship & $141 \mathrm{~K}^{*} 141 \mathrm{~K}$ & 7813404 & 28 \\
\hline & Economics & $207 \mathrm{~K}^{*} 207 \mathrm{~K}$ & 1273389 & 6 \\
\hline & Epidemiology & $526 \mathrm{~K}^{*} 526 \mathrm{~K}$ & 2100225 & 4 \\
\hline & FEM/Accelerator & $121 \mathrm{~K}^{*} 121 \mathrm{~K}$ & 2620000 & 22 \\
\hline & Circuit & $171 \mathrm{~K}^{*} 171 \mathrm{~K}$ & 958936 & 6 \\
\hline & Webbase & $1 \mathrm{M}^{*} 1 \mathrm{M}$ & 3105536 & 3 \\
\hline & LP & $4 \mathrm{~K} * 1.1 \mathrm{M}$ & 11279748 & 2825 \\
\hline & Circuit5M & $5.56 \mathrm{M}^{*} 5.56 \mathrm{M}$ & 59524291 & 11 \\
\hline & eu-2005 & $863 \mathrm{~K} * 863 \mathrm{~K}$ & 19235140 & 22 \\
\hline & Ga41As41H72 & $268 \mathrm{~K} * 268 \mathrm{~K}$ & 18488476 & 67 \\
\hline & in-2004 & $1.38 \mathrm{M}^{*} 1.38 \mathrm{M}$ & 16917053 & 12 \\
\hline & mip1 & $66 \mathrm{~K}^{*} 66 \mathrm{~K}$ & 10352819 & 152 \\
\hline & Si41Ge41H72 & $186 \mathrm{~K}^{*} 186 \mathrm{~K}$ & 15011265 & 81 \\
\hline
\end{tabular}

[Bell and Garland 2009], clSpMV [Su and Keutzer 2012], and CSR5 [Liu and Vinter 2015] for performance comparisons. CUSPARSE supports three formats: HYB, BCSR, and CSR. We manually searched the best performing configuration for each matrix. For the BCSR format in CUSPARSE, we also searched the block size for the best performance. For clSpMV, besides the COCKTAIL format that uses different formats for different partitions of a matrix, we also tested all the single formats and chose the best performing one for each matrix. Since the CUDA code is not supported on AMD platforms, we only compared our scheme with clSpMV on AMD FirePro W8000. On Intel MIC, we compared our scheme with CSR5 [Liu and Vinter 2015]. The code of our proposed framework is available at http://code.google.com/p/yaspmv/.

\section{EXPERIMENTAL RESULTS}

\subsection{Memory Footprint Size Comparison between Different Formats}

We evaluate the impact of our proposed $\mathrm{BCCOO} / \mathrm{BCCOO}+$ format on memory bandwidth. In BCCOO/BCCOO+ format, all the information, including the bit flag array, the col_index array, the data value array, and the auxiliary information described in Section 2.4, is only read once. We assume that it is also the case for all the other formats for comparison. Therefore, we can simply use the total size of the arrays to show the memory footprint of each format. The results are shown in Table IV. As our autotuning framework selects the BCCOO+ format only for the matrix LP, we do not separate the $\mathrm{BCCOO}$ and the $\mathrm{BCCOO}+$ formats. For some sparse matrices, due to the high variance in the number of nonzeros in different rows, the ELL format is not applicable (labeled "N/A" in Table IV). From Table IV, we find that BCCOO/BCCOO+ format significantly reduces the storage size of various sparse matrices. $\mathrm{BCCOO} / \mathrm{BCCOO}+$ format reduces the storage size by $40 \%$ on average compared with the COO format, $31 \%$ on average compared with the best single format among all the nine formats in clSpMV, and $21 \%$ on average compared with the COCKTAIL format. 
Table IV. The Memory Footprint Size (MB) of Different Formats

\begin{tabular}{|l|c|c|c|c|c|}
\hline Name & COO & ELL & Cocktail & Best Single & BCCOO \\
\hline \hline Dense & 48 & 32 & 17 & 17 & 17 \\
\hline Protein & 52 & 59 & 40 & 34 & 21 \\
\hline FEM/Spheres & 72 & 54 & 52 & 51 & 31 \\
\hline FEM/Cantilever & 48 & 39 & 25 & 25 & 21 \\
\hline Wind Tunnel & 140 & 314 & 78 & 78 & 65 \\
\hline FEM/Harbor & 28 & 54 & 24 & 24 & 14 \\
\hline QCD & 23 & 15 & 15 & 15 & 9 \\
\hline FEM/Ship & 94 & 115 & 56 & 59 & 34 \\
\hline Economics & 15 & 73 & 14 & 28 & 8 \\
\hline Epidemiology & 25 & 17 & 17 & 17 & 14 \\
\hline FEM/Accelerator & 31 & 79 & 26 & 25 & 17 \\
\hline Circuit & 12 & 483 & 9 & 23 & 6 \\
\hline Webbase & 37 & N/A & 29 & 138 & 27 \\
\hline LP & 135 & 1927 & 91 & 91 & 85 \\
\hline Circuit5M & 714 & N/A & 578 & 714 & 516 \\
\hline eu-2005 & 231 & N/A & 248 & 209 & 159 \\
\hline Ga41As41H72 & 222 & 1505 & 139 & 170 & 136 \\
\hline in-2004 & 203 & N/A & 209 & 203 & 132 \\
\hline mip1 & 124 & N/A & 66 & 54 & 51 \\
\hline Si41Ge41H72 & 180 & 983 & 118 & 135 & 105 \\
\hline Average & 122 & N/A & 93 & 106 & 73 \\
\hline
\end{tabular}

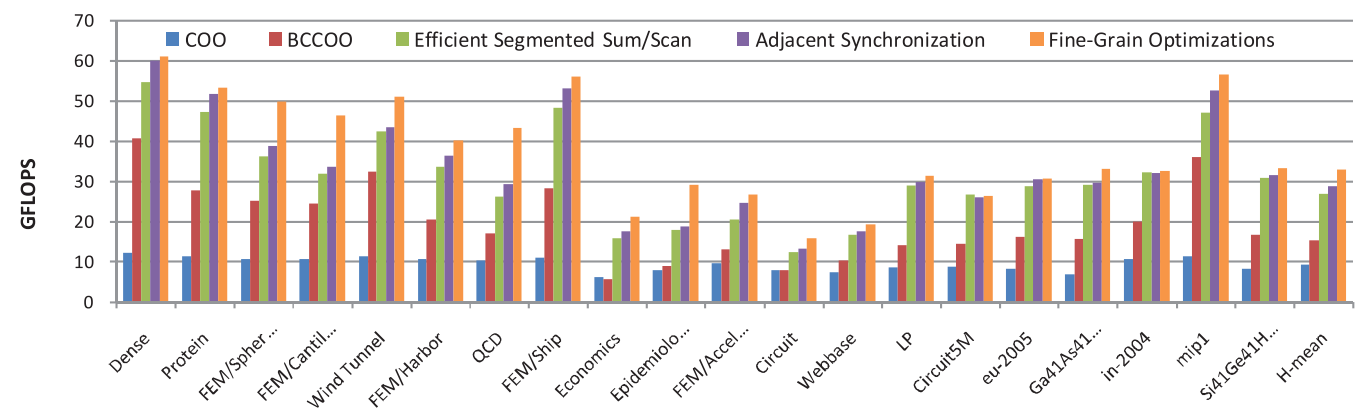

Fig. 13. Performance contributions from different optimization techniques on GTX680 (in single precision).

\subsection{Performance on NVIDIA and AMD GPUs}

We first examine the performance contributions from different optimizations in our approach, including memory footprint reduction, efficient segmented sum/scan, adjacent synchronization to remove global synchronization, and fine-grain optimizations, which consist of (a) the use of the short data type for the col_index array and (b) skip the parallel scan on a last_partial_sums array if possible. The results are shown in Figure 13. We start with the COO format with a tree-based segment sum (labeled "COO"). Then, we replace the COO format with our BCCOO/BCCOO+ format (labeled "BCCOO"). Next, we replace the tree-based segmented sum with our proposed efficient matrix-based segment sum/scan (labeled "Efficient segmented sum/scan"), in which the global synchronization is used to accumulate partial sums across workgroups. We then replace the global synchronization by the adjacent synchronization (labeled "adjacent synchronization") and add the fine-grain optimizations (labeled "fine-gain optimizations"). From the figure, we can see that the main performance gains are from our proposed BCCOO/BCCOO+ format and our efficient segmented sum/scan for SpMV. 


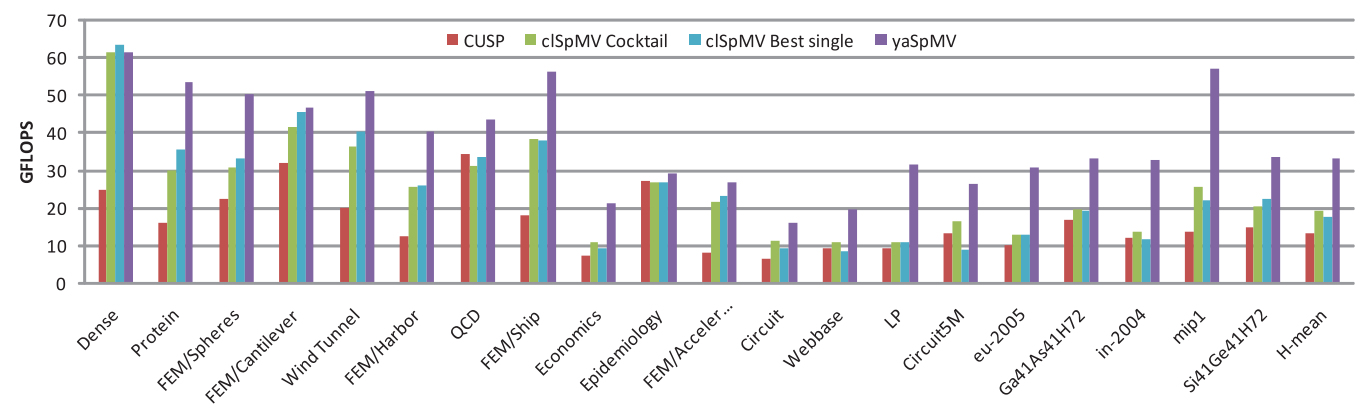

Fig. 14. Single-precision performance comparison between our proposed scheme (labeled "yaSpMV") and CUSP, clSpMV-best single, and clSpMV-COCKTAIL on GTX680 GPUs.

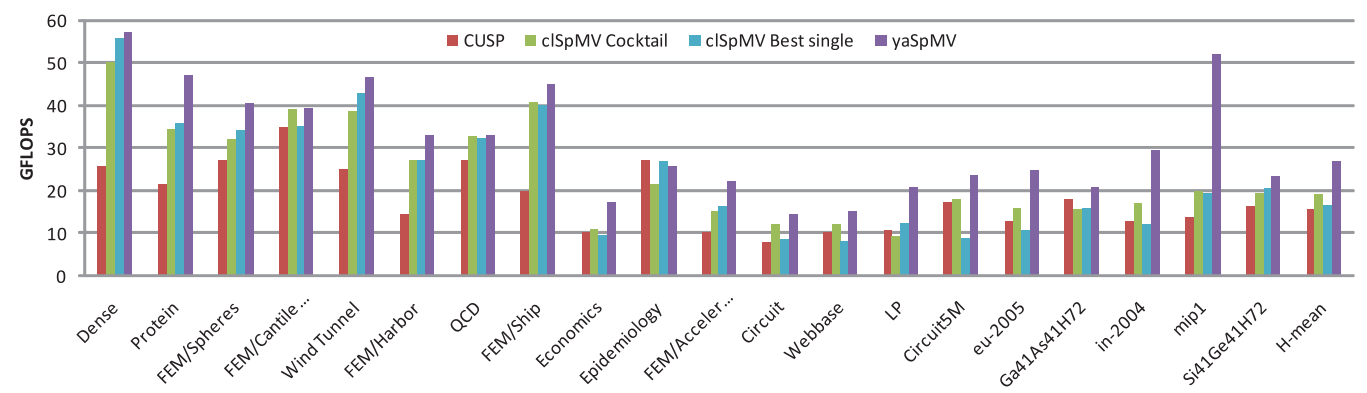

Fig. 15. Single-precision performance comparison between our proposed scheme (labeled "yaSpMV") and CUSP, clSpMV-best single, and clSpMV-COCKTAIL on GTX480 GPUs.

Next, we compare the performance of our proposed scheme with the state-of-theart techniques on GPUs. The single-precision performance on GTX680 is shown in Figure 14, in which our approach is labeled by "yaSpMV." We can see that yaSpMV outperforms the existing schemes for all the matrices except Dense. The Dense matrix prefers a block size of $2 \times 8$ in the BCSR format, which is selected as the best single format of clSpMV for Dense matrix. However, our autotuning framework limits the maximal block height to four, thereby achieving suboptimal performance. Using the harmonic mean (H-mean) as the average throughput, yaSpMV achieves an average performance improvement of $65 \%$ over CUSPARSE, $70 \%$ over clSpMV COCKTAIL, $88 \%$ over clSpMV best single, and $150 \%$ over CUSP. The highest performance gain of yaSpMV over clSpMV COCKTAIL is achieved on matrix LP (195\%). Compared with CUSPARSE, the highest performance gain of yaSpMV is achieved on matrix mip1 $(229 \%)$.

We further evaluate the single-precision performance of SpMV on Nvidia GTX480 GPUs, Tesla K20, GeForce Titan X, and AMD FirePro W8000 GPUs. The results are shown in Figures 15, 16, 17, and 18. On Nvidia GTX 480, our proposed yaSpMV achieves significantly higher performance than existing approaches (up to $162 \%$ better than clSpMV COCKTAIL and up to $150 \%$ better than CUSPARSE), as shown in Figure 15. The only exception is the Epidemiology matrix. It has four nonzeros on each row, which is a perfect fit for the ELL format. For this matrix, yaSpMV has a suboptimal performance of 25.5 GFLOPS. The best performing approach for this matrix, CUSPARSE, has a throughput of 28.5 GFLOPS. On average using H-mean, yaSpMV achieves a performance improvement of $40 \%$ over clSpMV COCKTAIL, $60 \%$ over clSpMV best single, $74 \%$ over CUSP, and $42 \%$ over CUSPARSE. As shown in Figures 16 and 17, yaSpMV achieves the performance improvement of $65.8 \%$ on average on Tesla K20 and $73.7 \%$ 


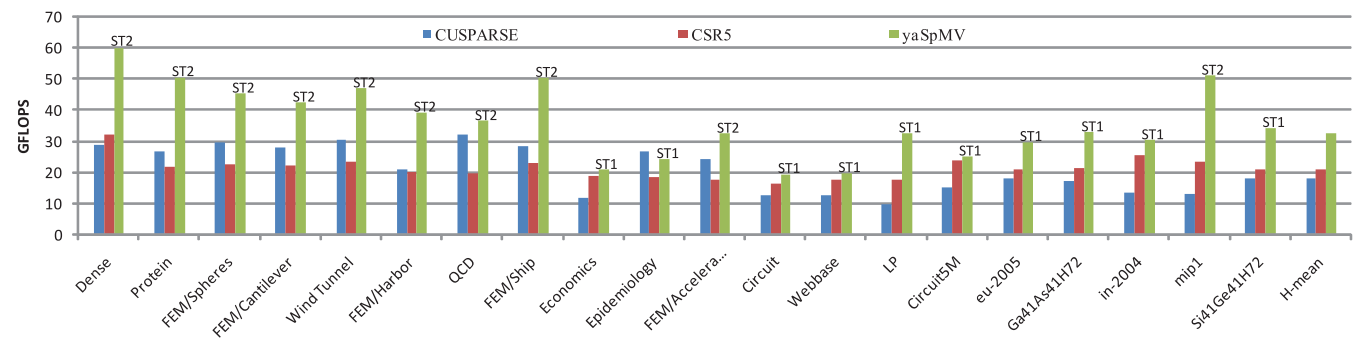

Fig. 16. Single-precison performance comparison between our proposed scheme (labeled "yaSpMV") and CUSPARSE V7.0, and CSR5 on Nvidia Tesla K20. We also mark the performance winner between strategy (1) (labeled "ST1") and strategy (2) (labeled "ST2") for yaSpMV.

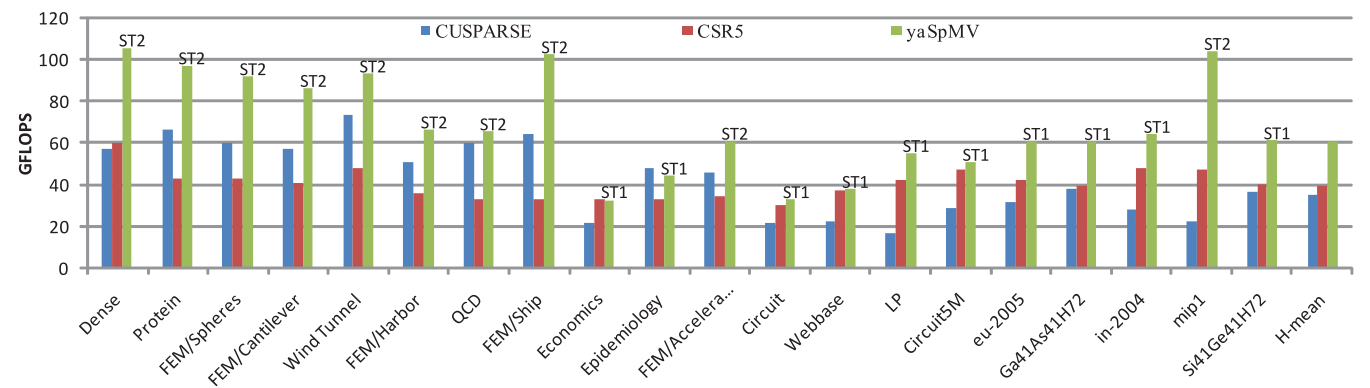

Fig. 17. Single-precison performance comparison between our proposed scheme (labeled "yaSpMV") and CUSPARSE V7.0, and CSR5 on GeForce Titan X GPUs. We also mark the performance winner between strategy (1) (labeled "ST1") and strategy (2) (labeled "ST2") for yaSpMV.

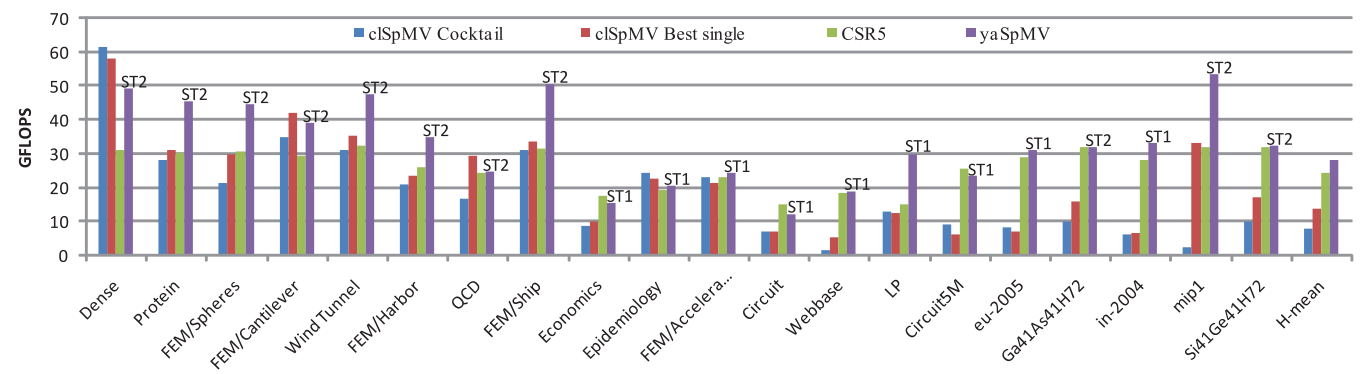

Fig. 18. Single-precision performance comparison between our proposed scheme (labeled "yaSpMV") and clSpMV-best single, clSpMV-COCKTAIL, and CSR5 on AMD FirePro W8000 GPUs. We also mark the performance winner between strategy (1) (labeled "ST1") and strategy (2) (labeled "ST2") for yaSpMV.

on average on GeForce Titan X over CUSPARSE V7.0; and achieves the performance improvement of $56.4 \%$ on average on Tesla K20 and $53.6 \%$ on average on GeForce Titan $\mathrm{X}$ over the recently proposed format-CSR5.

As shown in Figure 18, on AMD FirePro W8000, yaSpMV performs better than clSpMV COCKTAIL format on most matrices, and the performance improvement is up to $2617 \%$ and on average $255 \%$. Although there are only nine matrices for which our proposed yaSpMV performs better than the clSpMV best single format, yaSpMV also achieves a performance gain of $40 \%$ on average. Compared with CSR5, yaSpMV achieves a performance gain of $14.9 \%$ on average.

To further understand how strategy (1) and strategy (2) of yaSpMV perform on Tesla K20, GeForce Titan X, and AMD FirePro W8000, we mark the performance winner 


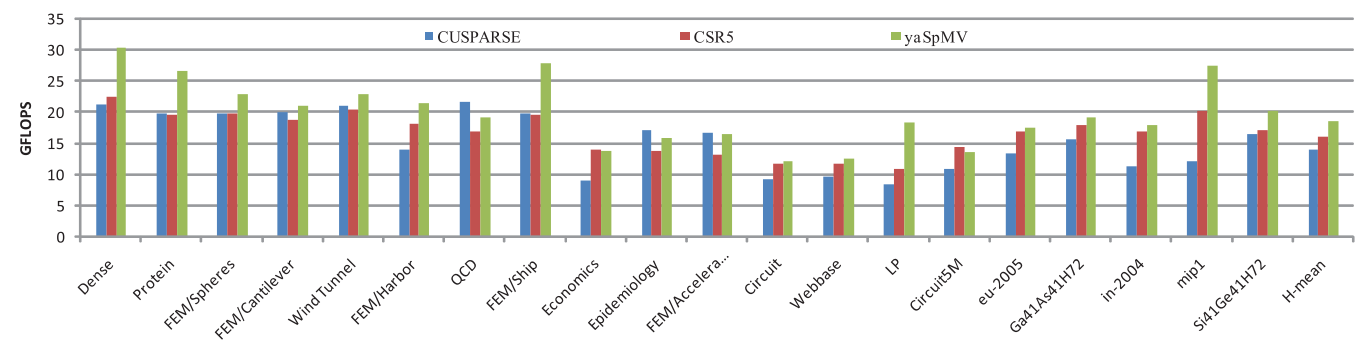

Fig. 19. Double-precison performance comparison between our proposed scheme (labeled "yaSpMV") and CUSPARSE V7.0, and CSR5 on Nvidia Tesla K20.

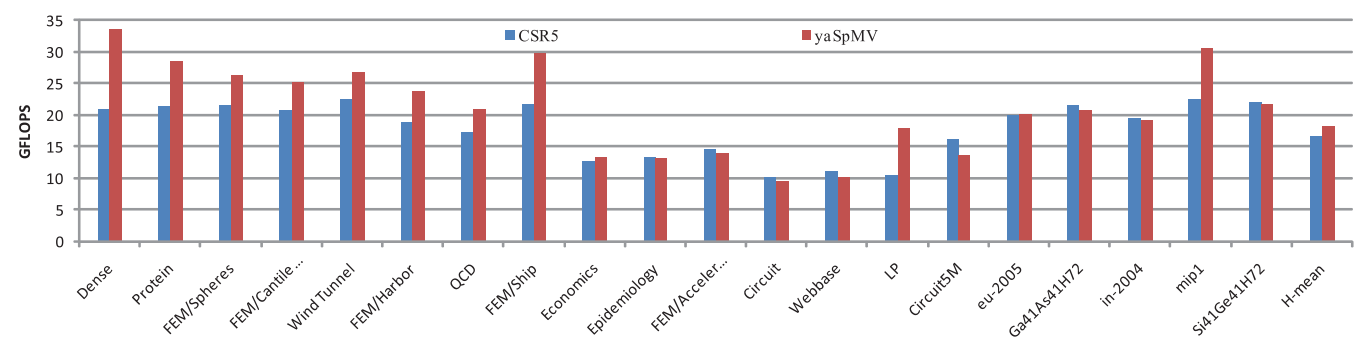

Fig. 20. Double-precision performance comparison between our proposed scheme (labeled "yaSpMV") and clSpMV-best single, clSpMV-COCKTAIL, and CSR5 on AMD FirePro W8000 GPUs.

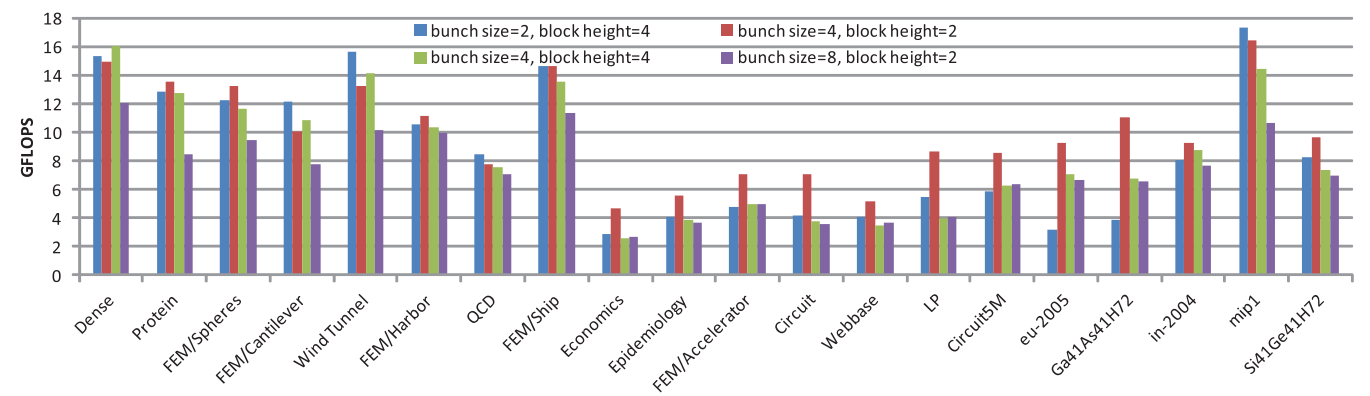

Fig. 21. Double-precision performance of our proposed scheme with different "bunch size" and "block height" on Intel MIC.

between these two strategies in Figures 16, 17, and 18. We find that both strategies are the potential winner for different sparse matrices, which demonstrates the necessity of the coexistence of the two strategies in our scheme.

We also evaluate the double-precision performance of SpMV on Tesla K20 and AMD FirePro W8000 GPUs. The results are shown in Figures 19-22. As shown in Figure 19, on Tesla K20, yaSpMV achieves the performance improvement of $34.0 \%$ on average over CUSPARSE V7.0 and $16.2 \%$ on average over CSR5. As shown in Figure 20, on AMD FirePro W8000, yaSpMV achieves the performance improvement of $9.7 \%$ on average over CSR5.

\subsection{Performance on Intel MIC}

Figure 21 presents the results of our proposed scheme with different "bunch size" and "block height" on Intel MIC. We can see that under different configurations of these two parameters, the performance varies largely. This is because the multiplication of these two parameters determines the width of SIMD instructions on Intel MIC, as 


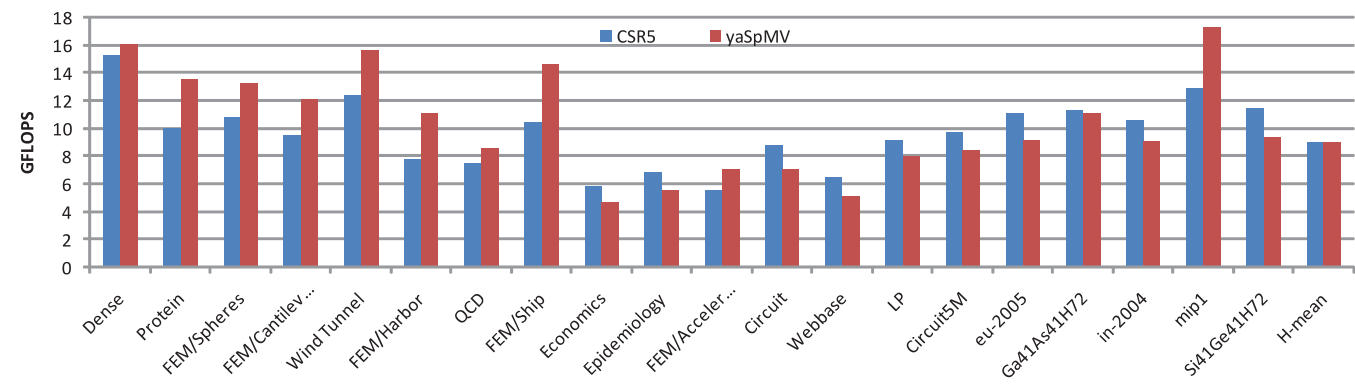

Fig. 22. Double-precision performance comparison between our proposed scheme (labeled "yaSpMV") and CSR5 on Intel MIC.

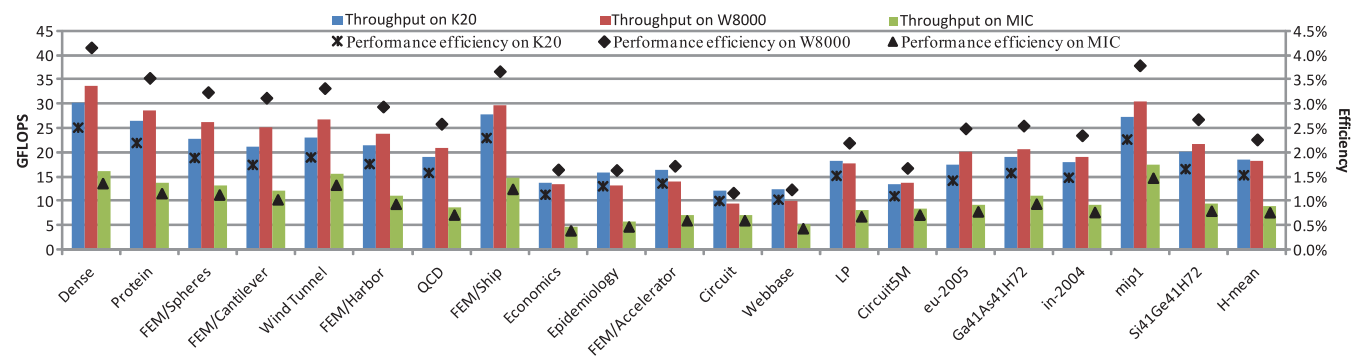

Fig. 23. Double-precision performance of yaSpMV on Nvidia Tesla K20, AMD FirePro W8000, and Intel MIC.

discussed in Section 3.2.2. When it fits to the width of 512 bits with less zero elements (determined by the value of "block height"), it can get the best performance. Thus, we need the autotuning framework to select the best configuration. As shown in Figure 22, the autotuned yaSpMV gets an average of 8.97 Gflops for the sparse matrixes on Intel MIC in double precision, which is comparable with CSR5 (an average of 9.01 Gflops).

We also compare the performance between GPUs (Nvidia Tesla K20 and AMD FirePro W8000) and Intel MIC in the context of yaSpMV, and the results is presented in Figure 23. Note that the double-precision peak performance of Nvidia Tesla K20, AMD FirePro W8000, and Intel MIC is 1.17 Tflops, 806 Gflops, and 1.011 Tflops, respectively. We find that both the throughput and the performance efficiency of GPUs are higher than Intel MIC. The average performance efficiency of yaSpMV on Nvidia K20, AMD W8000, and Intel MIC is $1.54 \%, 2.27 \%$, and $0.82 \%$, respectively. The lower efficiency of yaSpMV on Intel MIC is caused by the following reasons: (1) BCCOO is a blockbased format to exploit the register reusing. However, register blocking leads to low SIMD efficiency on Intel MIC [Liu et al. 2013]. Although we coarsen the workload of each thread to improve the SIMD efficiency, it still brings some performance penalty; (2) Intel MIC is lack of texture cache, which is more useful for the irregular accesses of the multiplied vector. Thus, the load imbalance of each thread caused by irregular accesses is more severe on Intel MIC.

\section{RELATED WORK}

Bolz et al. first introduced the GPUs for SpMV [Bolz et al. 2003]. Bell and Garland [2009] implemented several well-known formats on Nvidia GPUs. These formats include DIA, ELL, CSR, COO, and a hybrid format HYB, which combines the advantage of the ELL and COO formats. Su and Keutzer [2012] proposed the COCKTAIL format, which uses different formats to represent different partitions of a matrix. Vázquez 
et al. [2011] proposed a derivative format of ELLPACK, ELL-R. They use an auxiliary array to store the row lengths. Monakov et al. [2010] proposed the Sliced ELL format (SELL). They horizontally partition the original matrix into several slices to reduce the filling zeros. Compared with the ELL format, the ELL-R and SELL formats have less padding zeros. Kreutzer et al. [2014] proposed SELL-C- $\sigma$, which is a variant of SELL. To further reduce the padding overhead, the rows are sorted by the number of nonzero entries within a "sorting scope" $\sigma$. SELL-C- $\sigma$ is SIMD-friendly, and suitable for different architectures, including GPU, Intel MIC, and CPU. Compared with SELLC- $\sigma$, yaSpMV exploits register reusing and may have less padding zeros. However, the block-based design of yaSpMV makes it less suitable for Intel MIC than SELL-C- $\sigma$. We did not compare the performance of yaSpMV with SELL-C- $\sigma$, since the source code is not available to us yet. Liu et al. [2013] proposed the ESB format for Intel MIC, which extends the ELLPACK format with finite-window sorting for high SIMD efficiency, a bit array to encode nonzero locations for lower padding overhead, and column blocking for good locality. The column blocking method used in ESB motivates us to propose the $\mathrm{BCCOO}+$ format to improve the locality when accessing the multiplied vector.

Based on the CSR format, Koza et al. [2012] proposed a compressed multiple-row storage format for SpMV on GPUs. The advantage of this format is that the adjacent rows may be processed by the same thread, so the multiplied vector data could be reused. Choi et al. implemented the BCSR and BELL formats on GPUs [Choi et al. 2010] and proposed an autotuning framework. CSR-Adaptive [Greathouse and Daga 2014] is proposed to exploit the performance of SpMV with CSR format on GPUs. We tried to compare the performance of yaSpMV with CSR-Adaptive, which is implemented in ViennaCL. However, loading the sparse matrix from the disk to memory in ViennaCL is too time consuming (more than several days for some large matrices). Thus, we terminated the experiments on this format. Daga and Greathouse [2015] further improved CSR-Adaptive using novel reduction techniques and proposed a new SpMV algorithm for the irregular matrices with very long rows. Liu et al. presented CSR5 [Liu and Vinter 2015], which is insensitive to the sparsity structure of the input matrix and features fast format convention. Compared with CSR5, yaSpMV has almost equivalent performance on Intel MIC, and has an advantage on NVIDIA and AMD GPUs. Liu and Schmidt [2015] proposed LightSpMV, which uses the standard CSR format and improves the performance by fine-grained dynamic distribution of matrix rows over warps and vectors.

There are some works focusing on compression and reordering techniques as well [Buluç et al. 2011; Pichel et al. 2012]. The challenge of compression technique is the complexity of the decompression algorithm. The problem with the reordering technique is that it changes the inherent locality of the original matrix. A recent work by Tang et al. [2013] studies bit representations to compress index arrays. Similar to our work, a difference function is applied to index arrays. The difference from our proposed formats is that a bit packing scheme is then used to encode the delta values, which makes their decompression scheme more complicated than ours and also does not exploit the row stop information.

Blelloch et al. [1993] first introduced the segmented operations to SpMV on vector multiprocessors. Harris et al. [2007] implemented the segmented scan-based SpMV in the library CUDPP. Because they used a tree-based scan algorithm, which has been shown to be inefficient [Yan et al. 2013], the performance is limited. Baskaran and Bordawekar [2008] implemented a more efficient segmented scan-based SpMV using the matrix-based scan [Dotsenko et al. 2008]. However, their scan-based implementation also is outperformed by their alternative implementations [Baskaran and Bordawekar 2008]. Bell and Garland implemented their COO format using the segmented reduction (scan) algorithm. However, due to the disadvantage of the COO 
format and the two-kernel implementation, the performance is not highly competitive. Different from the previous works, we propose the new BCCOO/BCCOO+ format to drastically reduce the bandwidth requirement and efficient segmented $\mathrm{sum} / \mathrm{scan}$ algorithms on different many-core architectures. Our algorithm only needs one kernel and explores a number of optimization techniques.

\section{CONCLUSIONS}

In this article, we present yet another framework for SpMV on many-core architectures, including GPUs and Intel MIC. First, we propose a new format, called blocked compressed common coordinate (BCCOO), for sparse matrices. The key idea is to extend the $\mathrm{COO}$ format with blocking and to use a bit flag array to replace the row index array. We also propose to vertically partition a sparse matrix before using the BCCOO format, for better locality of the accesses to the multiplied vector. Second, we propose a highly efficient matrix-based segmented sum/scan for SpMV. Our matrix-based segmented $\mathrm{sum} / \mathrm{scan}$ is closely coupled to our BCCOO/BCCOO+ format to reduce the memory bandwidth and achieve load balance. Third, we propose an autotuning framework to further improve the performance with low overhead.

Our performance results from a set of 20 sparse matrices show that our proposed framework significantly advances the state-of-the-art SpMV schemes. In single precision, yaSpMV outperforms CUSPARSE 7.0 by $65.8 \%$ on average on Tesla K20, and by $73.7 \%$ on average on GeForce Titan X; outperforms clSpMV COCKTAIL format by $40 \%$ on average on GTX480 GPUs, by 70\% on average on GTX680 GPUs, and by $255 \%$ on average on AMD FirePro W8000 GPUs; and outperforms CSR5 by $56.4 \%$ on average on Tesla K20, by $53.6 \%$ on average on GeForce Titan X, and by $14.9 \%$ on average on AMD FirePro W8000. In double precision, yaSpMV outperforms CUSPARSE V7.0 by $34.0 \%$ on average on Tesla K20; and outperforms CSR5 by $16.2 \%$ on average on Tesla K20, and by $9.7 \%$ on average on AMD FirePro W8000. On Intel MIC, yaSpMV has almost equivalent performance compared with CSR5.

\section{ACKNOWLEDGMENTS}

The authors would like to thank the Supercomputing Center of CAS for providing free Intel MIC machines, and also thank Dr. Weifeng Liu from Niels Bohr Institute for his valuable advice on the performance evaluation of CSR5.

\section{REFERENCES}

Muthu Manikandan Baskaran and Rajesh Bordawekar. 2008. Optimizing sparse matrix-vector multiplication on GPUs using compile-time and run-time strategies. IBM Reserach Report, RC24704 (W0812-047) (2008).

Nathan Bell and Michael Garland. 2009. Implementing sparse matrix-vector multiplication on throughputoriented processors. In Proceedings of the Conference on High Performance Computing Networking, Storage and Analysis. ACM, 18.

Guy E. Blelloch. 1989. Scans as primitive parallel operations. IEEE Transactions on Computers 38, 11 (1989), 1526-1538.

Guy E. Blelloch, Michael A. Heroux, and Marco Zagha. 1993. Segmented Operations for Sparse Matrix Computation on Vector Multiprocessors. Technical Report. DTIC Document.

Jeff Bolz, Ian Farmer, Eitan Grinspun, and Peter Schröoder. 2003. Sparse matrix solvers on the GPU: Conjugate gradients and multigrid. In ACM Transactions on Graphics (TOG), Vol. 22. ACM, 917-924.

Ayd $\iota n$ Buluç, Samuel Williams, Leonid Oliker, and James Demmel. 2011. Reduced-bandwidth multithreaded algorithms for sparse matrix-vector multiplication. In Proceedings of the 2011 IEEE International Parallel \& Distributed Processing Symposium (IPDPS). IEEE, 721-733.

Jee W. Choi, Amik Singh, and Richard W. Vuduc. 2010. Model-driven autotuning of sparse matrix-vector multiply on GPUs. In ACM Sigplan Notices, Vol. 45. ACM, 115-126. 
Mayank Daga and Joseph L. Greathouse. 2015. Structural agnostic SpMV: Adapting CSR-adaptive for irregular matrices. In Proceedings of the 2015 IEEE 22nd International Conference on High Performance Computing (HiPC). IEEE, 64-74.

Timothy A. Davis and Yifan Hu. 2011. The University of Florida sparse matrix collection. ACM Transactions on Mathematical Software (TOMS) 38, 1 (2011), 1.

Yuri Dotsenko, Naga K. Govindaraju, Peter-Pike Sloan, Charles Boyd, and John Manferdelli. 2008. Fast scan algorithms on graphics processors. In Proceedings of the 22nd Annual International Conference on Supercomputing. ACM, 205-213.

Joseph L. Greathouse and Mayank Daga. 2014. Efficient sparse matrix-vector multiplication on GPUs using the CSR storage format. In SC14 Proceedings of the International Conference for High Performance Computing, Networking, Storage and Analysis. IEEE, 769-780.

Mark Harris, John Owens, Shubho Sengupta, Yao Zhang, and Andrew Davidson. 2007. CUDPP: CUDA data parallel primitives library. (2007).

Zbigniew Koza, Maciej Matyka, Sebastian Szkoda, and Łukasz Mirosław. 2012. Compressed Multiple-row Storage Format. Technical Report.

Moritz Kreutzer, Georg Hager, Gerhard Wellein, Holger Fehske, and Alan R. Bishop. 2014. A unified sparse matrix data format for efficient general sparse matrix-vector multiplication on modern processors with wide SIMD units. SIAM Journal on Scientific Computing 36, 5 (2014), C401-C423.

ShiGang Li, ChangJun Hu, JunChao Zhang, and YunQuan Zhang. 2015. Automatic tuning of sparse matrixvector multiplication on multicore clusters. Science China Information Sciences 58, 9 (2015), 1-14.

Weifeng Liu and Brian Vinter. 2015. Csr5: An efficient storage format for cross-platform sparse matrix-vector multiplication. In Proceedings of the 29th ACM on International Conference on Supercomputing. ACM, 339-350.

Xing Liu, Mikhail Smelyanskiy, Edmond Chow, and Pradeep Dubey. 2013. Efficient sparse matrix-vector multiplication on x86-based many-core processors. In Proceedings of the 27th International ACM Conference on International Conference on Supercomputing. ACM, 273-282.

Yongchao Liu and Bertil Schmidt. 2015. LightSpMV: Faster CSR-based sparse matrix-vector multiplication on CUDA-enabled GPUs. In Proceedings of the 2015 IEEE 26th International Conference on ApplicationSpecific Systems, Architectures and Processors (ASAP). IEEE, 82-89.

Alexander Monakov, Anton Lokhmotov, and Arutyun Avetisyan. 2010. Automatically tuning sparse matrixvector multiplication for GPU architectures. In High Performance Embedded Architectures and Compilers. Springer, 111-125.

CUDA NVIDIA. 2014. CUSPARSE library. (2014). NVIDIA Corporation, Santa Clara, California.

Juan C. Pichel, Francisco F. Rivera, Marcos Fernández, and Aurelio Rodríguez. 2012. Optimization of sparse matrix-vector multiplication using reordering techniques on GPUs. Microprocessors and Microsystems 36,2 (2012), 65-77.

Shubhabrata Sengupta, Mark Harris, Yao Zhang, and John D. Owens. 2007. Scan primitives for GPU computing. In Graphics Hardware, Vol. 2007. 97-106.

John E. Stone, David Gohara, and Guochun Shi. 2010. OpenCL: A parallel programming standard for heterogeneous computing systems. Computing in Science \& Engineering 12, 1-3 (2010), 66-73.

Bor-Yiing Su and Kurt Keutzer. 2012. clSpMV: A cross-platform OpenCL SpMV framework on GPUs. In Proceedings of the 26th ACM International Conference on Supercomputing. ACM, 353-364.

Wai Teng Tang, Wen Jun Tan, Rajarshi Ray, Yi Wen Wong, Weiguang Chen, Shyh-hao Kuo, Rick Siow Mong Goh, Stephen John Turner, and Weng-Fai Wong. 2013. Accelerating sparse matrix-vector multiplication on GPUs using bit-representation-optimized schemes. In Proceedings of the International Conference on High Performance Computing, Networking, Storage and Analysis. ACM, 26.

Sain-Zee Ueng, Melvin Lathara, Sara S. Baghsorkhi, and W. Hwu Wen-mei. 2008. CUDA-lite: Reducing GPU programming complexity. In Languages and Compilers for Parallel Computing. Springer, 1-15.

Francisco Vázquez, José-Jesús Fernández, and Ester M. Garzón. 2011. A new approach for sparse matrix vector product on NVIDIA GPUs. Concurrency and Computation: Practice and Experience 23, 8 (2011), $815-826$.

Samuel Williams, Leonid Oliker, Richard Vuduc, John Shalf, Katherine Yelick, and James Demmel. 2009. Optimization of sparse matrix-vector multiplication on emerging multicore platforms. Parallel Comput. 35, 3 (2009), 178-194.

Shengen Yan, Guoping Long, and Yunquan Zhang. 2013. StreamScan: Fast scan algorithms for GPUs without global barrier synchronization. In ACM SIGPLAN Notices, Vol. 48. ACM, 229-238.

Received February 2016; revised August 2016; accepted August 2016 